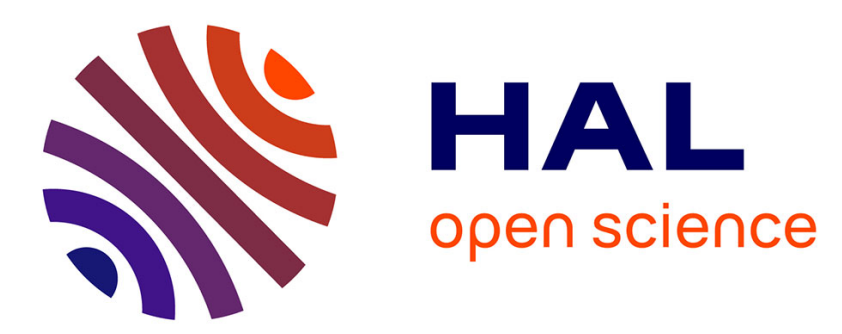

\title{
A decision tool for disassembly process planning under end-of-life product quality
}

\author{
Mohand Lounes Bentaha, Alexandre Voisin, Pascale Marangé
}

\section{To cite this version:}

Mohand Lounes Bentaha, Alexandre Voisin, Pascale Marangé. A decision tool for disassembly process planning under end-of-life product quality. International Journal of Production Economics, 2020, 219, pp.386-401. 10.1016/j.ijpe.2019.07.015 . hal-02315859

\section{HAL Id: hal-02315859 \\ https://hal.univ-lyon2.fr/hal-02315859}

Submitted on 25 Oct 2021

HAL is a multi-disciplinary open access archive for the deposit and dissemination of scientific research documents, whether they are published or not. The documents may come from teaching and research institutions in France or abroad, or from public or private research centers.
L'archive ouverte pluridisciplinaire HAL, est destinée au dépôt et à la diffusion de documents scientifiques de niveau recherche, publiés ou non, émanant des établissements d'enseignement et de recherche français ou étrangers, des laboratoires publics ou privés.

\section{(ㄷ)(1) $\$$}

Distributed under a Creative Commons Attribution - NonCommercial| 4.0 International 


\title{
A decision tool for disassembly process planning under End-of-Life product quality
}

\author{
Mohand-Lounes Bentaha ${ }^{\mathrm{a}, *}$, Alexandre Voisin ${ }^{\mathrm{b}}$, Pascale Marangéb \\ ${ }^{a}$ Université Lyon 2, DISP Laboratory (EA 4570), Lyon, France \\ ${ }^{b}$ Université de Lorraine, CRAN, UMR CNRS 7039, Campus Sciences, \\ BP 7023954506 Vandœuvre-lès-Nancy Cedex, France
}

\begin{abstract}
One of the major sources of uncertainty in disassembly systems is the quality or states of postconsumer products. This paper develops a decision tool for disassembly process planning under variability of the End-of-Life product quality. The objective is to maximize the profit of the disassembly process. This latter is calculated as the difference between the revenue generated by recovered parts and the cost of the disassembly tasks. The revenue of a product (or a subassembly, or a component) depends on its quality. The proposed methodology helps to take decisions about the best disassembly process and the depth of disassembly, depending on the quality of the products to be disassembled. Industrial applicability and interest are shown using an industrial case focused on the remanufacturing of mechatronic parts in the automotive industry.

Keywords: sustainable manufacturing, product recovery, disassembly, quality uncertainty management, decision support system
\end{abstract}

\section{Introduction}

Reverse logistics and its processes are nowadays well accepted and understood. Indeed, at the European level, the Brundtland report [1] and worldwide, the United Nations Conference on Sustainable Development are some examples of such concern. This leads to consider a more sustainable consumption model considering, instead of "use and dispose", "use and re-use". Such model is called circular [2] as disposed equipments are considered as reusable. Moreover, as pointed out in

\footnotetext{
*Corresponding author

Email addresses: mohand.bentaha@univ-lyon2.fr (Mohand-Lounes Bentaha), alexandre.voisin@univ-lorraine.fr (Alexandre Voisin), pascale.marange@univ-lorraine.fr (Pascale Marangé)
} 
[3], ease materials recycling, reuse and re-manufacturing constitute critical factors for sustainable competitiveness. Besides its environmental advantages among which a raw material and energy saving of $80 \%-90 \%$ on average [4], it allows the creation of highly skilled jobs and economic growth [5] and the saving of disposal cost that manufacturers must support by law. Furthermore, the remanufacturing market is intended for growth since it is under-evaluated and still receives little interest: the ratio of remanufacturing over new manufacturing is only $1.9 \%$ [5]. Indeed, remanufacturing generates around 30bn€ in turnover and employs around 190,000 people and projections could lead to 70bn€ to $90 \mathrm{bn} €$ in turnover with creation of jobs between 34,000 and 65,000 [5]. Finally, re-manufacturing is a key enabler technology $[6,7]$ not only for sustainable development but as well for economic and social developments. As such, it addresses the 3 pillars of sustainability, i.e. Economic, Social, and Environmental.

The process of re-manufacturing requires (1) to disassemble, (2) to clean, (3) to inspect, diagnose and sort, (4) to re-condition and (5) to re-assemble $[4,8]$. When considering "systems", with several sub-systems as cars, computers, etc., a prior step to disassembly is required in order to diagnose the defect and remaining functionalities [9]. Such prior step is a pre-require since returned products are subject to highly variable condition [4]. The main causes for such a variability in returned product condition are the age, the customer use modes and the environmental condition the product suffered. Moreover, the lack of product traceability, in turn, results in the fact lack of knowledge on their quality. The lack of reliable information for re-manufacturing leads to opportunities being missed with respect to increased economic or environmental impact [3]. It leads to uncertainty and high variability in the disassembly step while other steps remain less impacted. The impact on disassembly mainly includes technical issues in disassembly and variability of the processing time [10]. Therefore, on one hand, the prior inspection step is of first importance since it will decide the disassembly "level", and, on the other hand, it is hard to perform since inspection and diagnostic of in-situ sub-systems, while they are still mounted on the system, is challenging. Hence, disassembly lines remain artisanal with versatile workstations at the expense of efficiency. In order to consider disassembly at an industrial level, one has to tackle these variability and uncertainty in returned product quality. 
The present paper considers the disassembly process as an industrial process. In such a way, a single type of product is considered as imput flow of the disassembly process. Such hypothesis seems realistic when considering mass products such as automobiles, cell phones, laptops, refrigerators, etc. Hence, the disassembly process cannot be considered as an artisanal-work manner as is currently. Thus, disassembly process has to be planned in advance and its financial viability has to be demonstrated.

The aim of this work is to contribute to such a goal. It proposes a tool in order to define the optimal disassembly depth/level of a product regarding the profit. The originality of the proposed approach is to consider the health state of the product, its parts and sub-parts in the revenue estimation as random variables. The state of a part allows to decide its re-cycling: maintenance, re-use, regeneration or raw material recycling [11]. The re-cycling of a product impacts in a nonlinear way its resale price. The recycling decision requires a quantification of the part capacity to re-enter a cycle. For such a purpose, we introduce the Remaining Usage Potential (RUP).

The economical optimization of the disassembly process considers the cost of the disassembly tasks and the revenue of the resale of the disassembled parts $[12,13]$. The latter is highly dependent on the re-entering usage cycle whose decision is based on the RUP. The RUPs of a product and its parts are considered as distributions since we consider mass recycling.

This paper is structured as follows. A literature review of disassembly planning, taking into account the quality of products, is presented in Section 2. This is followed in Section 3 by a formal description of the studied problem. Section 4 presents the developed model along with the solution approach. Numerical experiments and optimization results are presented in Section 5. Section 6 concludes the paper with future research directions.

\section{Literature review}

In the literature, the work dealing with disassembly process planning can be classified into two categories: disassembly sequence planning and disassembly line design and balancing. Here, disassembly sequence planning includes work where execution order of disassembly tasks is explicitly mentioned as well as work where the execution order is not considered. The first category is studied in many papers among which we can cite [10, 14-19]. A review paper on the subject is presented 
in [20]. Nevertheless, very few studies have dealt with the case of presence of uncertainty. Among these works, a study on disassembly process planning under uncertainty of the whole process duration is presented in [21]. Studies in [22] and [23] considered the sequence dependent disassembly process planning. In other words, how to determine the disassembly sequence where task processing times depend on their execution order. In [23], the sequence dependent disassembly process planning is studied along with the disassembly line balancing problem. [24] and [25] studied the problem of disassembly process planning under uncertainty of product parts quality. The quality of a product part is considered as a discrete state. Both papers used Petri Nets as a modeling tool. In [24], two quality classes are considered for each product part: repairable or worn out. In [25], several quality classes are defined as degradation levels. In addition, the revenue generated from each state, in both papers, is a predetermined constant value. There is no explicit relation between a quality state and the gained revenue.

In the studies of the second category, disassembly process planning constitute a subproblem. In fact, the problem consists of selecting a disassembly process and assigning the corresponding tasks to a number of workstations which is determined simultaneously. In [26], the authors considered the disassembly line balancing and sequencing under uncertainty of the task processing times. Even for this category, disassembly process planning under uncertainty remains far less explored compared to the deterministic case. Almost all the papers were restricted to the consideration of uncertainty of the disassembly task processing times. We can cite [27-29]. In particular, the work in [26, 30-34] proposes mathematical models and exact solution approaches to select the best disassembly process alternative and determine a first design of a disassembly line, under uncertainty of the task processing times. However, variability caused by the End-of-Life product states (quality) was not taken into account explicitly. A summary of these different papers is presented in [35]. Studies in [36, 37] considered the case of task failures caused by defective parts of an End-of-Life product. A comprehensive survey on disassembly line design and balancing can be found in [38-40].

Recently, attempts to include End-of-Life quality in disassembly process planning have been made in [4, 41]. In [41], disassembly process planning for disassembly line balancing problem 
under complete product disassembly and multiple product quality classes was proposed. In [4], the concept of quality classes adapted from [41] was used considering, this time, partial disassembly for line balancing. Task processing times for each class were assumed to be random variables with known non truncated normal probability distributions. This approach of task uncertainty modeling along with the proposed solution approach are adapted from [32]. In [4], the End-of-Life product quality is considered implicitly in the same manner as in [32]. The only difference is the fact that products are classified on different quality categories and then, for each quality class, the method adapted from [32] is applied. In addition, both in [41] and [4], the quality classes concern only entire End-of-Life product, ignoring the quality of their subassemblies and components generated during the disassembly process. Furthermore, these studies have not used and/or graphs to model the precedence relationships among tasks and subassemblies and components. Koc et al. [42] showed that the integration of and/or graphs in the problem formulation allowed better solutions to be obtained compared to the use of simple and precedence diagrams. Also, most of the and/or graphs used in the literature are part based diagrams. Such graphs do not exploit the precedence relations among tasks and parts (subassemblies and components) of the End-of-Life product. As a matter of fact, a modeling process and methodology that are able to jointly define the best disassembly process alternative and the best disassembly level when disassembly revenue depends explicitly on the quality of post-consumer products, along with their sub-assemblies and components, has never been proposed.

One of the main goals of this paper is to answer this question: what is the economical impact of uncertain product quality on the disassembly process or strategy to be adopted in remanufacturing systems? This study proposes for the first time a decision tool based on a methodology to optimize the specific disassembly strategy and process considering quality of the input return products in the remanufacturing planning phase. And/or graphs are used to better model the precedence relations among disassembly tasks and product parts obtained during the disassembly process. The proposed methodology is applied to a product example, taken and adapted from [4], which represents a real case study in the automotive part remanufacturing sector, to show its industrial applicability and benefits. 


\section{Problem definition and modeling}

In this work, we consider a remanufacturing process where the revenue from retrieved parts (subassemblies and components) depends on the quality of the incoming return products. For an End-of-Life product, the problem consists on the selection of a best disassembly process alternative, among all possible ones, taking into account its RUP and precedence relationships amongst all disassembly tasks and product parts obtained during the disassembly process.

All possible disassembly process alternatives for an End-of-Life product are represented with a set $I$ of all the possible disassembly tasks of that product. The objective is to maximize the profit generated from the best disassembly process alternative to be determined. An optimal disassembly process alternative includes only a subset $I^{\prime}$ of the tasks $I$. The subset of disassembly tasks $I^{\prime}$ defines the depth of the disassembly process and it is known after the optimization process. This will be explained in detail in subsection 3.1.

The following assumptions are used. A single type End-of-Life product has to be partially (or completely) disassembled. All received items contain all initial parts with no addition or removing of components. In the case of industrialized disassembly, as it is for remanufacturing systems, this hypothesis seems realistic when a large percentage of products arrive in these conditions. The maximum disassembly level is the components level, tasks for material recycling of components after disassembly are not considered. This assumption corresponds to the components resale as raw materials. A fixed cost is assigned per disassembly time unit to each disassembly task. Each component or subassembly has a resale value which represents its revenue. This revenue depends on the quality of the corresponding component or subassembly. The state or quality of each subassembly is modeled using the concept of RUP which follows a probability distribution. This distribution models the variability of RUP of the input return products, as well as their subassemblies and components. In the optics of industrialized disassembly, i.e. a large number of products of the same category are returned, to obtain such a probability distribution, statistical studies on disassembled products can be conducted. The RUP concept is explained in detail in subsections 3.2, 3.3 and 3.4. 


\subsection{And/or graph}

The and/or graph, considered in this paper, represents explicitly all the possible disassembly process alternatives of an End-of-Life product along with the precedence relationships among tasks and the different product subassemblies and components.

In order to better explain in an intuitive manner the modeling process, we have chosen as a product a ball point pen taken from the literature [26]. This ball point pen is composed of 10 components, as shown in Figure 1. Components are identified by their respective numbers and the materials they are made of (cooper $(\mathrm{Cu})$, polypropylene $(\mathrm{PP})$, polyvinyl chloride (PVC), stainless steel, steel alloy and ink). The and/or graph in Figure 2 corresponds to the ball point pen example and is constructed as follows: each subassembly is represented by a node labeled $A_{k}, k \in K$. For example, node $A_{0}$ represents the ball point pen which can be noted as ' $1: 10$ ', $A_{1}$ represents the subassembly '1:3-5:10', etc. (see Table 1). For a simplification reason of the and/or graph, the components generated by all disassembly tasks are not explicitly represented in the graph, only the subassemblies are. Set $K$, then, contains only the indices of all possible subassemblies that can be generated by all the disassembly tasks. Each node labeled $B_{i}, i \in I$, represents a disassembly task. For instance, node $B_{1}$ represents disassembly task '1', $B_{2}$ represents disassembly task '2', etc. Set $I$ defines all the possible disassembly tasks. Table 1 summarizes all the possible disassembly tasks that can be performed on the ball point pen in Figure 1. For each task, the corresponding generated subassemblies and components are given.

Two types of arcs define the precedence relations between subassemblies (or components) and disassembly tasks: and and or. The first type imposes a mandatory precedence relation and the second type is employed for optional precedence dependencies, where only one option is chosen for a final solution. For example, if a disassembly task generates two subassemblies or more (as

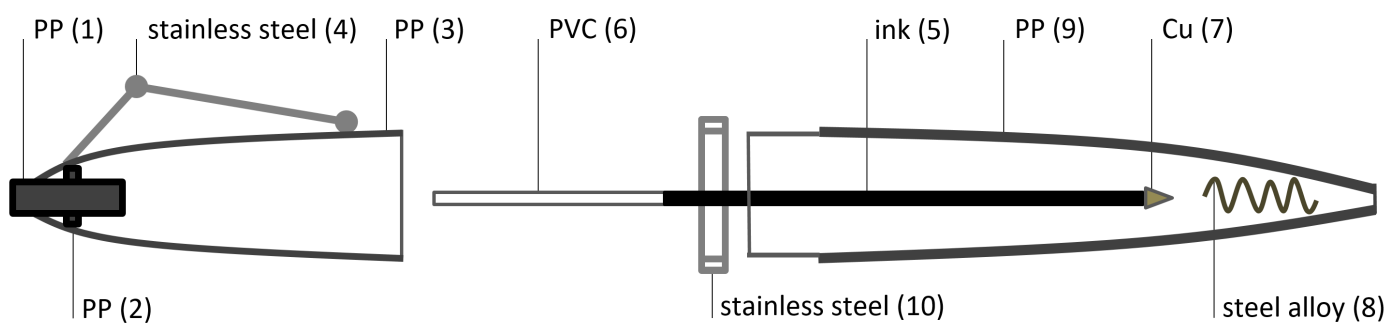

Figure 1: A ball point pen product example 


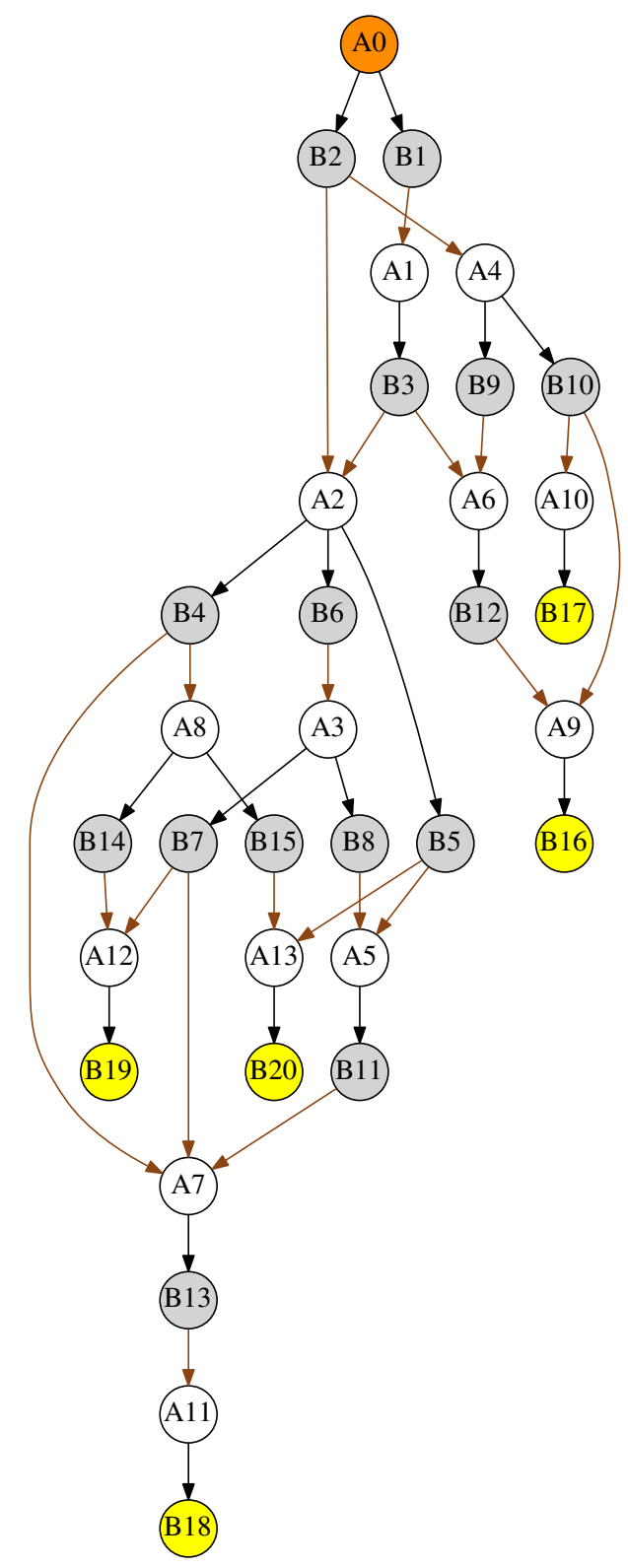

Figure 2: The and/or graph of the ball point pen example

$B_{10}$ generates $A_{10}$ and $A_{9}$ ), then it is related to these subassemblies by and-type arcs (in brown color in Figure 2). If several concurrent disassembly tasks may be performed on a subassembly (as subassembly $A_{3}$ can be disassembled by $B_{7}$ or $B_{8}$ ), this subassembly is related to these disassembly tasks by or-type arcs.

Table 1 can be operated as follows: the number of all possible disassembly tasks is 20 ; this defines $I$. The total number of subassemblies and components which can be generated with complete 
Table 1: Ball point pen associated tasks and corresponding subassemblies and components

\begin{tabular}{|c|c|c|c|c|c|}
\hline$x^{25}$ & $\sqrt{a^{v^{2}}}$ & & $x^{0^{3}}$ & 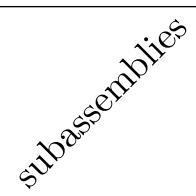 & $v^{2}$ \\
\hline 1 & $A_{1}(1: 3-5: 10)$ & 4 & 11 & $A_{7}(5: 7)$ & 8 \\
\hline 2 & $A_{2} ; A_{4}(5: 10 ; 1: 4)$ & - & 12 & $A_{9}(1-2)$ & 3 \\
\hline 3 & $A_{2} ; A_{6}(5: 10 ; 1: 3)$ & - & 13 & $A_{11}(5-6)$ & 7 \\
\hline 4 & $A_{7} ; A_{8}(5: 7 ; 8: 10)$ & - & 14 & $A_{12}(8-9)$ & 10 \\
\hline 5 & $A_{5} ; A_{13}(5: 8 ; 9-10)$ & - & 15 & $A_{13}(9-10)$ & 8 \\
\hline 6 & $A_{3}(5: 9)$ & 10 & 16 & - & $1 ; 2$ \\
\hline 7 & $A_{7} ; A_{12}(5: 7 ; 8-9)$ & - & 17 & - & $3 ; 4$ \\
\hline 8 & $A_{5}(5: 8)$ & 9 & 18 & - & $5 ; 6$ \\
\hline 9 & $A_{6}(1: 3)$ & 4 & 19 & - & $8 ; 9$ \\
\hline 10 & $A_{9} ; A_{10}(1-2 ; 3-4)$ & - & 20 & - & $9 ; 10$ \\
\hline
\end{tabular}

disassembly process from $I$ is 23 ; this defines $K$ and all generated components. The numbers 1 to 10 represent respectively the ball point pen components 1 to 10, see Figure 1. The numbers 11 to 23 symbolize respectively the different subassemblies generated by the corresponding tasks. The number representing $A_{1}$ is 11 , that of $A_{2}$ is 12 , that of $A_{3}$ is $13, \ldots$ (each time add 10 to the index of $A ; 10$ is here the components number of the ball point pen). Reading example of Table 1: task $B_{2}$ disassembles $A_{0}$ (the ball point pen) and generates two subassemblies (Figure 2): subassembly $A_{2}$ composed of components $5,6,7,8,9$ and 10 (represented with ' $5: 10$ ') and subassembly $A_{4}$ composed of components 1, 2, 3 and 4 (represented with '1:4'). Disassembly task $B_{2}$ does not generate any component. Disassembly task $B_{1}$ generates component 4 and subassembly $A_{1}$, which is composed of components $1,2,3,5,6,7,8,9$, and 10 .

A sink node ' $\mathrm{S}$ ' is introduced and linked with dummy arcs to all the disassembly tasks (Figure 3). The dummy arcs linking each disassembly task $B_{i}$ of $I$ to the node ' $\mathrm{S}$ ' allow the representation of the disassembly depth. Hence, partial disassembly, for each disassembly process alternative of the product, is modeled using the and/or graph in Figure 3.

Figure 4 illustrates a disassembly process alternative of the ball point pen. Typically, such a disassembly process alternative is returned by the decision tool, developed in this paper, after the optimization process. The disassembly process alternative shown in Figure 4 defines a partial disassembly of the product. In fact, for a complete disassembly, all the tasks constituting this 


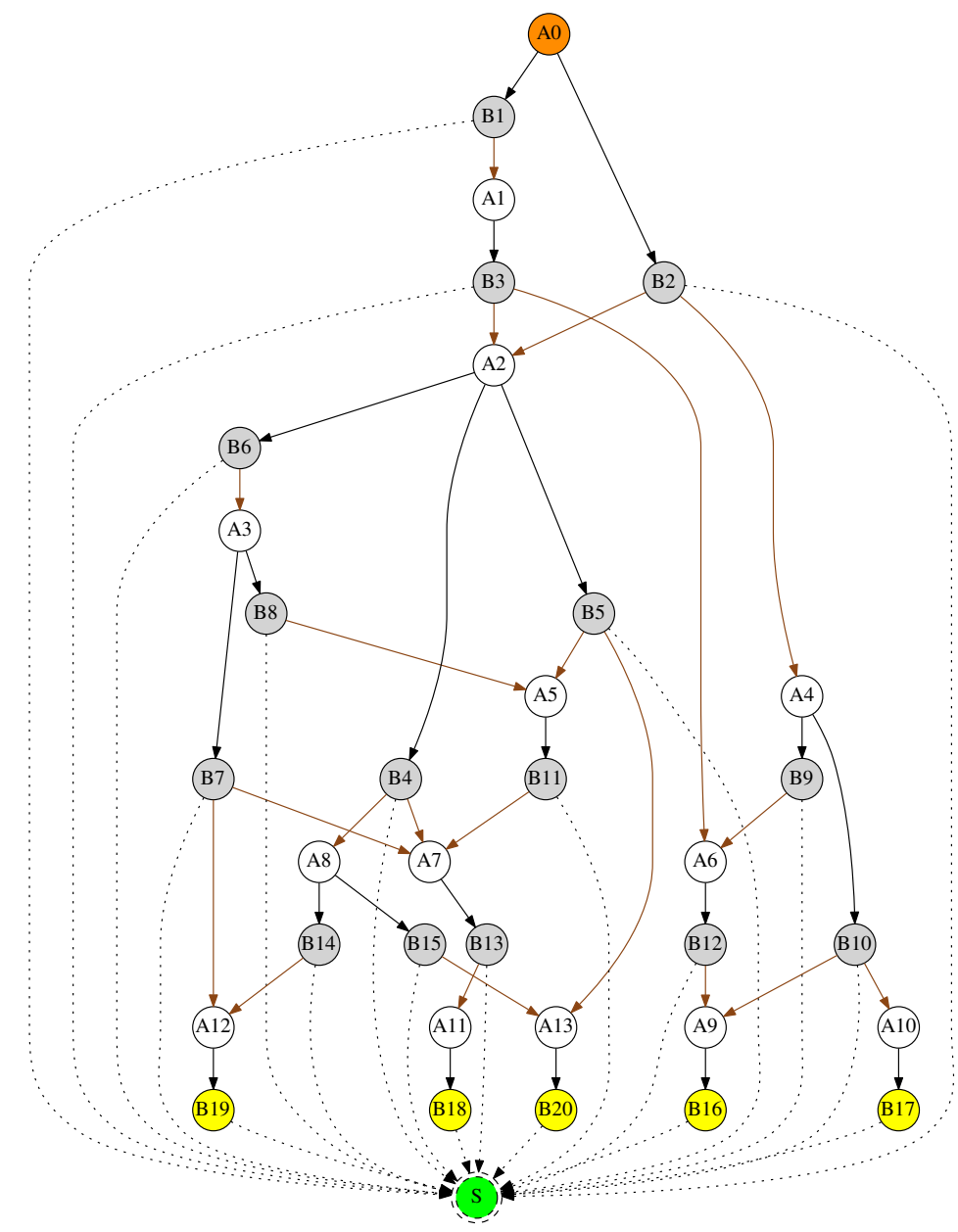

Figure 3: And/or graph of the ball point pen example allowing partial disassembly representation

disassembly alternative must be executed. There are 9 tasks in total: $B_{2}, B_{10}, B_{16}, B_{17}, B_{4}, B_{13}$, $B_{15}, B_{18}, B_{20}$. However, only 4 tasks are selected to define the disassembly process: $B_{2}, B_{4}, B_{13}$ and $B_{15}$, as shown in bold in Figure 4 . Set $I^{\prime}$ is defined by these 4 selected tasks.

The disassembly process defined by $I^{\prime}$ allows to retrieve subassemblies $A_{4}, A_{11}$ and $A_{13}$ (respectively 1:4, 5-6 and 9-10) and components 7 and 8. Since subassemblies $A_{4}, A_{11}$ and $A_{13}$ are no longer disassembled, then tasks $B_{13}$ and $B_{15}$ generating them are directly linked to the node $\mathrm{S}$ with dummy arcs, as illustrated in Figure 4. Table 1 gives, as cited earlier, for each disassembly task the retrieved subassemblies and components after its execution. 


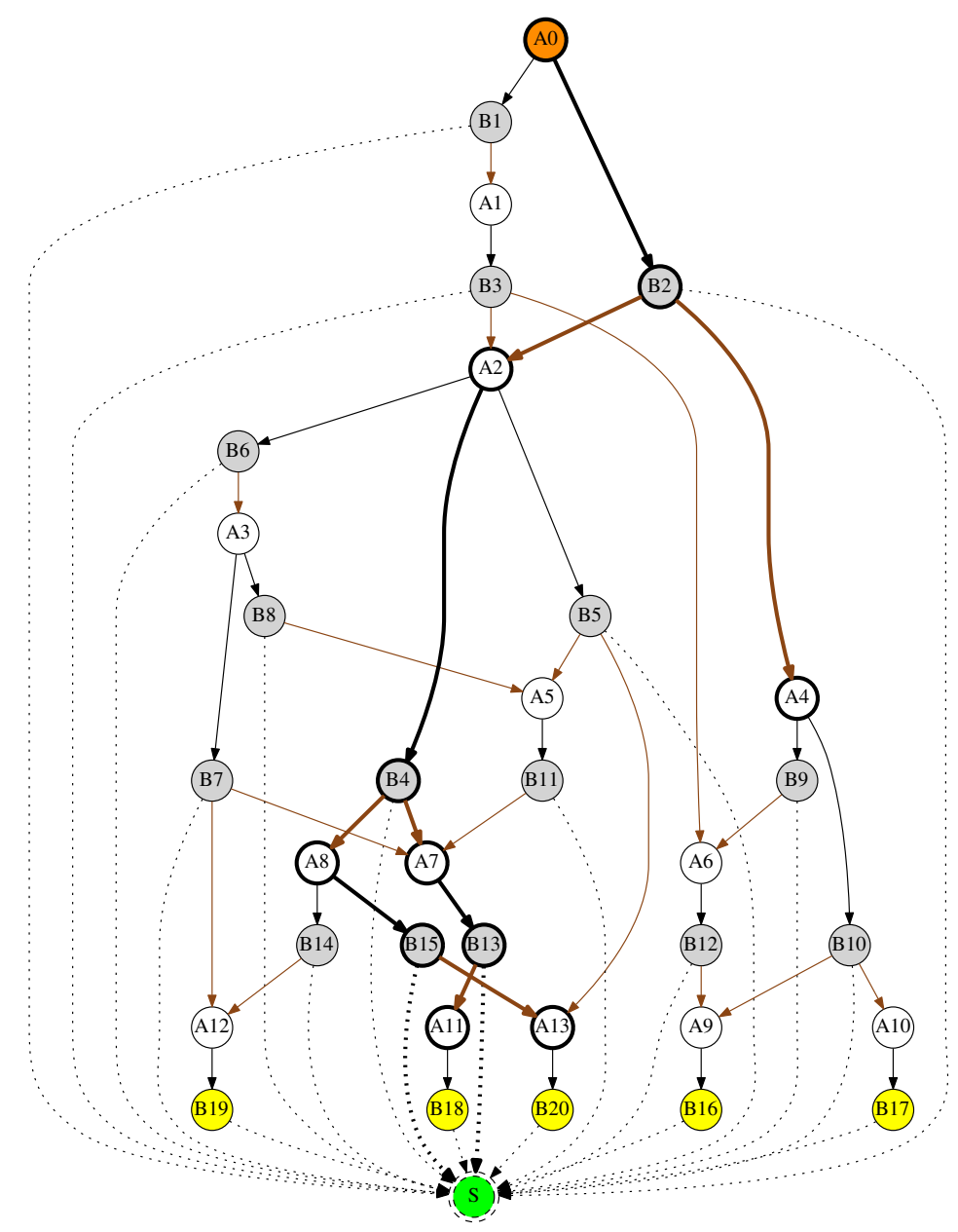

Figure 4: A selected disassembly alternative (partial disassembly)

\subsection{Remaining Usage Potential and its probability density function}

The main originality of the paper is to consider the state of the product for disassembly. Indeed, for a mature product, when it goes out of the assembly line, it has been designed to fulfill some reliability requirements, meaning that its components have been chosen in order to long for a defined time, in some usage condition and at a required probability. Hence, products at the beginning of their usage are in the same state. Obviously, in "real life" usage, the product encounters several situations and environments that it has not been designed for. Such usage leads to degradations and even failure. Maintenance allows to restore the product in a state such that it can perform its function but do not fully recover its initial state. Furthermore, the whole sub-components are not impacted in the same way. Thus, they have their own health state which differs from the global product. 
In order to assess the state to be considered for disassembly, we defined the Remaining Usage Potential (RUP). The RUP stands for the "usage" quantity remaining for a product or a part. In our proposition, the RUP is evaluated prior to the disassembly. The RUP is evaluated on a $[0,1]$ scale. 1 corresponds to the product state at the beginning of its exploitation (the product is at its maximal RUP); 0 means that the product/part has reached its end of life and has to be recycled as raw material.

The RUP concept is different from known concepts such as Remaining Useful Life (RUL) or reliability. Indeed, RUL and reliability assess the product ability to reach a certain time using either degradation prognostic or probability of performing a function. Both evaluate the product over "one" maintenance cycle, i.e. its ability to perform its function until the next maintenance. The RUP evaluates the ability of a product to perform its function over several maintenance cycles. Hence, it includes the product performance and its ability to maintain its use over a long period.

The usage of a product will make its RUP decreasing according to: (1) the environment, (2) the way the product is used, (3) the way the product is maintained and (4) the way the product is built and designed. These 4 factors influencing the RUP will necessarily differ for all products. They lead to a spread of the RUP when considering several products at the same usage time. Furthermore, this spread is increased since products do not arrive at the disposal at the same usage time.

Although the main subject of the paper is not the assessment of the RUP, the following directions shall be considered to be able to quantify it:

- within the reliability theory, one can consider the Beta parameter of the Weibull distribution [43]. Indeed, the Beta parameter is representative of the long term degradation of a system. Further developments of the Weibull distribution has been done in [44];

- within Prognostics and Health Management strategy $[45,46]$, the product is monitored along its whole lifetime [47]. The RUL is used in order to take the right decision at the right time. The RUL is provided thanks to the prognostic process. The RUL is the time left until a failure or a performance loss occurs. It allows the planning of the next maintenance action. The RUL differs from the RUP since its time horizon is restricted only to the next maintenance 
action while the RUP's one is at longer. In analogy with PHM of battery, the RUL could be seen as the state of charge (SoC) [48] and the RUP as the state of health (SoH) [49]. The monitored data shall be stored all along the usage life of the product such as proposed in the MIMOSA initiative ${ }^{1}$. Such a longitudinal monitoring could be used to evaluate the "disposal" state of a product;

- finally, if none of the two above apply, one can consider, as in the recycling of end of life products, to estimate the state of a product, since we consider mass product recycling, a statistical sampling which can be performed on a periodic basis. For instance, in France, Indra ${ }^{2}$ performs (re)qualification of automotive parts, Paperec ${ }^{3}$ and Remade $^{4}$ do the same in the WEEE. Such (re)qualification process leads to propose different levels of waranty according to the grade of the component.

Considering the "ball point pen" example (Figure 1), one can consider:

- the pen body (component 9) and the button (component 1) have been heavily used lead to a visible wear. Their reuse as such is unlikely leading to a low RUP;

- the spring (component 8) is heavily used as well. However, it often remains in a state good enough to be reused straight forwardly leading to a high RUP;

- the ring (component 10) and the clip (component 4) have a more random state since their usage is more customer related, leading to a spread RUP.

The RUP is modeled as a probability density function since this study considers a mass disassembly process. As such, the number of products to be considered is high and the RUP shall be considered in a statistical way. As this study is a first step, we consider the RUP as a normal probability density function truncated in 0 and 1 . The RUP follows a truncated normal distribution on $[0,1]$ with $\mu$ and $\sigma$ parameters: $\operatorname{RUP} \rightsquigarrow \mathcal{N}_{[0,1]}(\mu, \sigma) ; \mu$ and $\sigma$ are respectively the mean and standard deviation of the original non truncated normal distribution. The mean and standard

\footnotetext{
${ }^{1}$ http://www.mimosa.org/

${ }^{2}$ ww . indra.fr/en/home

3 WwW.paprec.com/en/solutions-and-services/recycling-solutions/batteries-and-weee

4 wW . remadegroup.com/en/know-how/
} 
deviation of the $[0,1]$ truncated normal distribution are given by:

$\mu_{[0,1]}=\mu+\frac{\phi\left(\frac{0-\mu}{\sigma}\right)-\phi\left(\frac{1-\mu}{\sigma}\right)}{\Phi\left(\frac{1-\mu}{\sigma}\right)-\Phi\left(\frac{0-\mu}{\sigma}\right)} \sigma$

$\sigma_{[0,1]}=\sigma \sqrt{1+\frac{\frac{0-\mu}{\sigma} \phi\left(\frac{0-\mu}{\sigma}\right)-\frac{1-\mu}{\sigma} \phi\left(\frac{1-\mu}{\sigma}\right)}{\Phi\left(\frac{1-\mu}{\sigma}\right)-\Phi\left(\frac{0-\mu}{\sigma}\right)}-\left(\frac{\phi\left(\frac{0-\mu}{\sigma}\right)-\phi\left(\frac{1-\mu}{\sigma}\right)}{\Phi\left(\frac{1-\mu}{\sigma}\right)-\Phi\left(\frac{0-\mu}{\sigma}\right)}\right)^{2}}$

where $\phi(x)=\frac{1}{\sqrt{2 \pi}} e^{-\frac{1}{2} x^{2}}$ is the standard normal probability density function and $\Phi(\cdot)$ its cumulative distribution function.

Figure 5 shows, over $[0,1]$ interval, 3 truncated normal distribution functions with parameters: $(\mu=0, \sigma=0.2),(\mu=0.5, \sigma=0.3)$ and $(\mu=1, \sigma=0.2)$. Curves in Figure 5 shall stand for the RUP of either a product, a part (component) or a sub-part (subassembly):

- the yellow curve, with $\mu=0$ and $\sigma=0.2$, shows a heavily worm part whose RUP is statistically low;

- the blue curve, with $\mu=1$ and $\sigma=0.2$, shows a slightly used part whose RUP is statistically high;

- the red curve in the middle, with $\mu=0.5$ and $\sigma=0.3$, shows a middle term part whose RUP is statistically average.

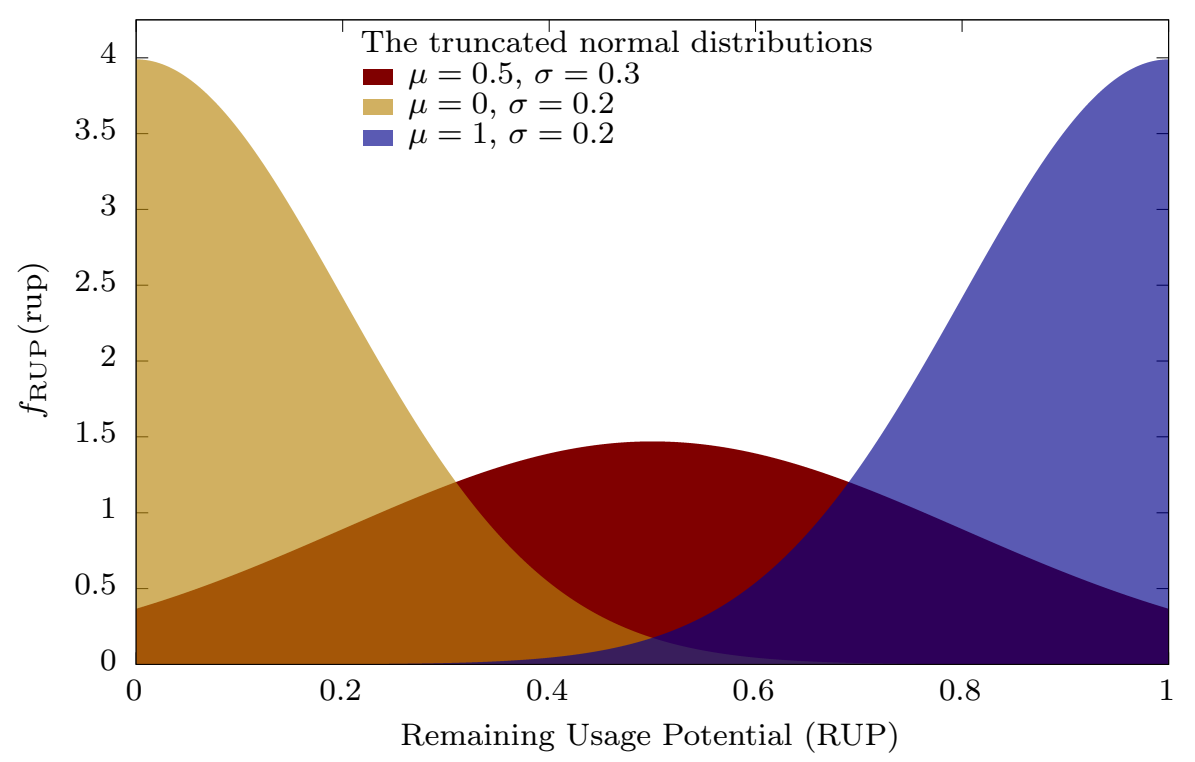

Figure 5: RUP distrbution examples following normal distributions truncated on 0 and 1 


\subsection{Part revenue with respect to its $R U P$}

Since the items have not the same RUP, their resale prices have to be considered as functions of their RUPs. The resale price represents the revenue $\left(R_{e}\right)$ due to recycling recovery of disassembly items. Obviously, the higher the RUP, the higher the revenue. The following notation is used to model the RUP:

\section{RUP : Remaining Usage Potential;}

$\mathcal{N}_{[0,1]}(\mu, \sigma)$ : truncated normal distribution on 0 and 1 with parameters $\mu$ and $\sigma$; in this paper, RUP follows such a probability density function, but any adequate probability distribution function can be used (see susection 3.2 for more details);

$\mu_{[0,1]}$ : mean of the $[0,1]$ truncated normal distribution given by: $\mu_{[0,1]}=\mu+\frac{\phi\left(\frac{0-\mu}{\sigma}\right)-\phi\left(\frac{1-\mu}{\sigma}\right)}{\Phi\left(\frac{1-\mu}{\sigma}\right)-\Phi\left(\frac{0-\mu}{\sigma}\right)} \sigma$; $\phi(\cdot)$ is the standard normal probability density function and $\Phi(\cdot)$ its cumulative distribution function;

$\sigma_{[0,1]}$ : standard deviation of the $[0,1]$ truncated normal distribution given by: $\sigma_{[0,1]}=\sigma \sqrt{1+\frac{\frac{0-\mu}{\sigma} \phi\left(\frac{0-\mu}{\sigma}\right)-\frac{1-\mu}{\sigma} \phi\left(\frac{1-\mu}{\sigma}\right)}{\Phi\left(\frac{1-\mu}{\sigma}\right)-\Phi\left(\frac{0-\mu}{\sigma}\right)}-\left(\frac{\phi\left(\frac{0-\mu}{\sigma}\right)-\phi\left(\frac{1-\mu}{\sigma}\right)}{\Phi\left(\frac{1-\mu}{\sigma}\right)-\Phi\left(\frac{0-\mu}{\sigma}\right)}\right)^{2}}$

$f_{\text {RUP }}($ rup $)$ : symbolizes the RUP probability density function (PDF) of a given disassembly item; $f_{\text {RUP }}(\operatorname{rup})=$ $\mathcal{N}_{[0,1]}(\mu, \sigma)$ of the considered disassembly item;

$R_{e}(\mathrm{RUP})$ : revenue due to recycling recovery of a disassembly item; $R_{e}$ is a function of the item RUP, written $R_{e}(\mathrm{RUP})$

$f_{R_{e}}\left(r_{e}\right)$ : symbolizes the revenue probability density function of a disassembly item; combining the RUP probability density function $f_{\mathrm{RUP}}(\operatorname{rup})$ with the item revenue function $R_{e}(\mathrm{RUP})$ gives the part revenue probability density function $f_{R_{e}}\left(r_{e}\right)$.

We assume that the resale price is bounded. The upper bound corresponds to the resale price of an "almost new" used item. Indeed, it is not realistic to consider the sale price as upper bound since as soon as an item is used it depreciates. The lower bound corresponds to the resale price of the raw material of the item. The upper bound, resp. the lower one, corresponds to $R_{e}(\mathrm{RUP}=1)=b$, 
resp. $R_{e}(\mathrm{RUP}=0)=a$, with $b>a>0$.

We defined, for illustration purpose, 3 cases for $R_{e}$ according to the RUP, with the corresponding definition of $R_{e}(\mathrm{RUP})$ :

- the first case considers a linear RUP revenue function, i.e. $R_{e}$ is proportional to the item's RUP:

$R_{e}=(b-a) \cdot \mathrm{RUP}+a$, affine function

- the second case considers a rapid growth of the $R_{e}$ revenue according to the item's RUP with a stabilization when the item's RUP becomes medium. Such a $R_{e}$ revenue variation is modeled with a square root function. Such a case means that the re-sale price of an item remains hight the long "RUP" term despite the drop of the RUP. Two root functions are considered for this case:

$R_{e}=(b-a) \cdot \sqrt{\mathrm{RUP}}+a$, square root function

$(\operatorname{root} 1)$

$R_{e}=(b-a) \cdot \sqrt[4]{\mathrm{RUP}}+a, 4^{\text {th }}$ root function

$($ root 2$)$

- the third one considers $R_{e}$ low for low and medium RUP levels and increases rapidly for high levels of the RUP:

$R_{e}=a e^{\ln \left(\frac{b}{a}\right) \cdot \operatorname{RUP}}$, exponential function

(expo 1)

$R_{e}=e^{\frac{1}{e-1}(e \ln (a)-\ln (b))} e^{\frac{1}{e-1}(\ln (b)-\ln (a)) e^{\mathrm{RUP}}}$, second exponential function

(expo 2)

Figure 6 illustrates, for $a=5$ and $b=50$, the 5 revenue functions. The RUP axis can be read from the right to the left. Indeed, such reading is the "natural" one since the RUP decreases as the item is used. The interpretation of the root and exponential cases are as follows:

- root function: the revenue decreases slowly despite the item is good or fair health state. Indeed, the item keeps a quite high resale price as long as it is at he beginning and until the middle of its life or as long as it is of good or fair quality. For poor health state, when the item reaches the end of its life, the revenue decreases rapidly.

- exponential function: the revenue decreases rapidly even if the item is of good quality or at 


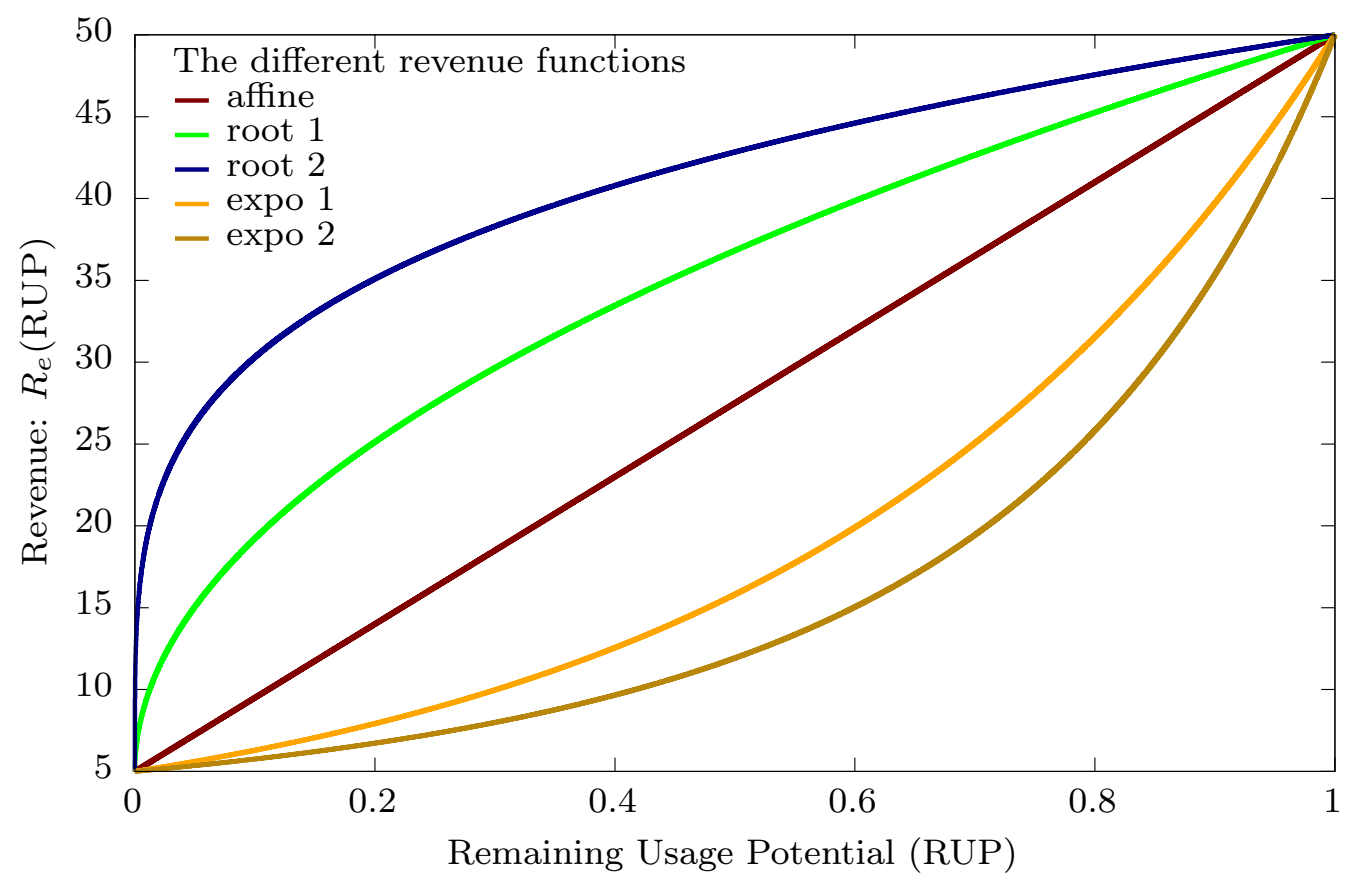

Figure 6: Different curves representing variations of $R_{e}$ function of RUP

the beginning of its life. Then, for fair to poor quality items, from the middle up to the end of its life, the item keeps a quite low resale price.

\subsection{Part revenue probability density function}

Combining the RUP probability density function with the part revenue function gives the part revenue probability density function. For the $5 R_{e}$ functions presented above, the corresponding probability density functions are:

$$
\begin{aligned}
f_{R_{e}}\left(r_{e}\right) & =\frac{1}{b-a} \frac{\phi\left(\mu, \sigma, \frac{r_{e}-a}{b-a}\right)}{\Phi(\mu, \sigma, 1)-\Phi(\mu, \sigma, 0)} \mathbb{I}_{[a, b]} \\
f_{R_{e}}\left(r_{e}\right) & =\frac{2}{(b-a)^{2}}\left(r_{e}-a\right) \frac{\phi\left(\mu, \sigma,\left(\frac{r_{e}-a}{b-a}\right)^{2}\right)}{\Phi(\mu, \sigma, 1)-\Phi(\mu, \sigma, 0)} \mathbb{I}_{[a, b]} \\
f_{R_{e}}\left(r_{e}\right) & =\frac{4}{(b-a)^{4}}\left(r_{e}-a\right)^{3} \frac{\phi\left(\mu, \sigma,\left(\frac{r_{e}-a}{b-a}\right)^{4}\right)}{\Phi(\mu, \sigma, 1)-\Phi(\mu, \sigma, 0)} \mathbb{I}_{[a, b]} \\
f_{R_{e}}\left(r_{e}\right) & =\frac{1}{r_{e} \ln \left(\frac{b}{a}\right)} \frac{\phi\left(\mu, \sigma, \frac{1}{\ln \left(\frac{b}{a}\right)} \ln \left(\frac{r_{e}}{a}\right)\right)}{\Phi(\mu, \sigma, 1)-\Phi(\mu, \sigma, 0)} \mathbb{I}_{[a, b]}
\end{aligned}
$$


$f_{R_{e}}\left(r_{e}\right)=\frac{1}{r_{e}\left(\ln \left(r_{e}\right)-\alpha\right)} \frac{\phi\left(\mu, \sigma, \ln \left(\frac{\ln \left(r_{e}\right)-\alpha}{\beta}\right)\right)}{\Phi(\mu, \sigma, 1)-\Phi(\mu, \sigma, 0)} \mathbb{I}_{[a, b]}$

where $\alpha=\frac{1}{e-1}(e \ln (a)-\ln (b))$ and $\beta=\frac{1}{e-1}(\ln (b)-\ln (a))$;

$e=2.71828 \cdots$ is the Euler's constant

$\phi(\mu, \sigma, x)=\frac{1}{\sigma \sqrt{2 \pi}} e^{-\frac{1}{2 \sigma^{2}}(x-\mu)^{2}}$ defines the normal probability density function of mean $\mu$ and standard deviation $\sigma$ and $\Phi(\mu, \sigma, \cdot)$ defines its cumulative distribution function.

We consider 3 cases for the RUP distribution, i.e. RUP $\rightsquigarrow \mathcal{N}_{[0,1]}(\mu, \sigma)$, according to the part quality (see Figure 5): bad $(\mu=0, \sigma=0.2)$, medium $(\mu=0.5, \sigma=0.3)$ and $\operatorname{good}(\mu=1, \sigma=0.2)$. Figures 7,8 and 9 show the probability density function $f_{R_{e}}\left(r_{e}\right)$ for $R_{e}$ according to the 3 RUP distribution cases and the 5 revenue functions.

In the case of bad quality parts, i.e. $\mu=0$ and $\sigma=0.2$, the average revenue in the case of the root functions is quite good, around 22 for root1 and 33 for root2, despite the bad state of the parts (see figure 7). On the contrary, when considering good quality parts, i.e. $\mu=1$ and $\sigma=0.2$, the average revenue in the case of exponential functions is quite low, around 28 for expo2 and 37 for expo1, despite the good state of the parts.

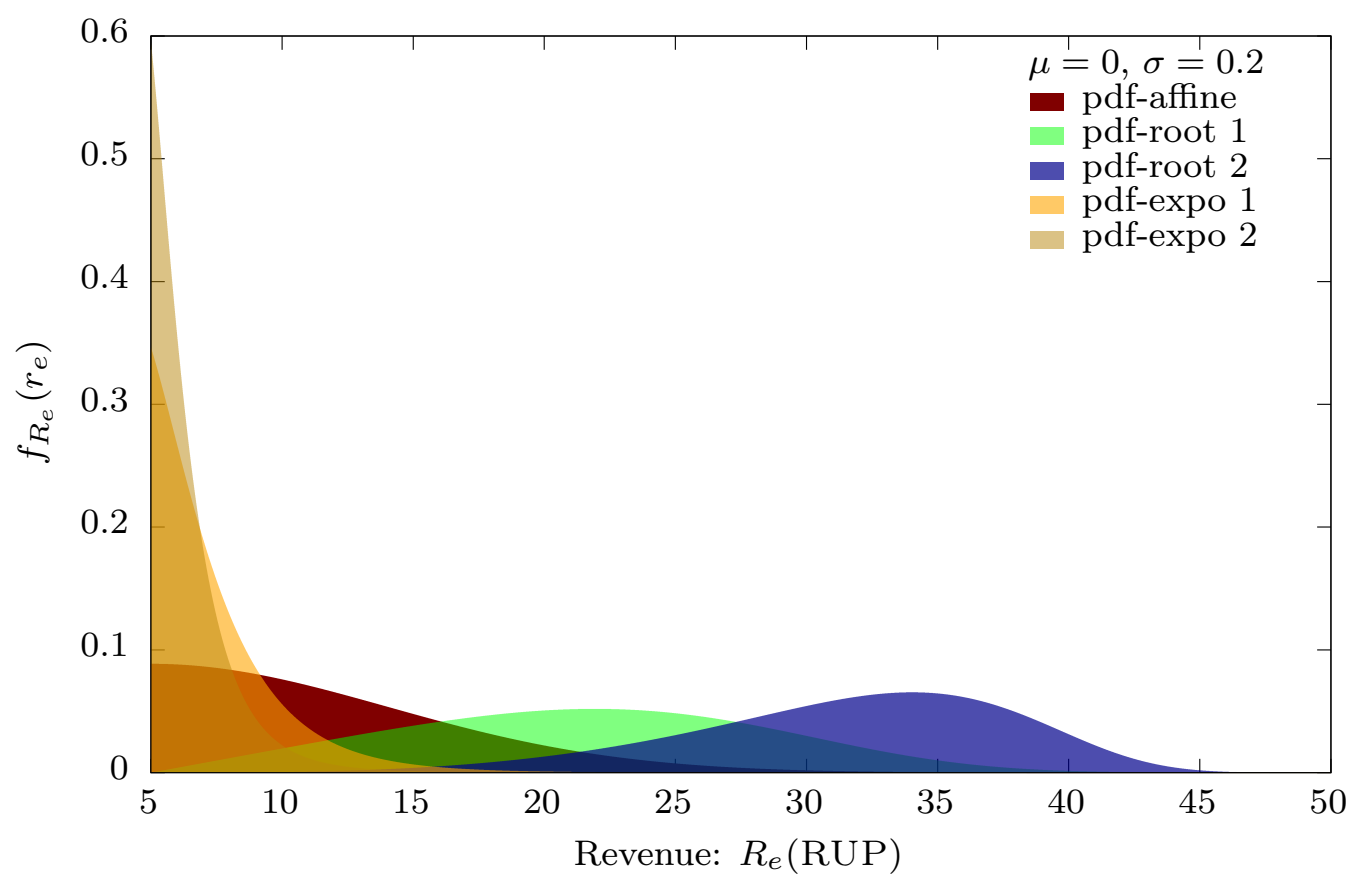

Figure 7: Different probability density functions of $R_{e}(\mathrm{RUP})$ : bad part quality 


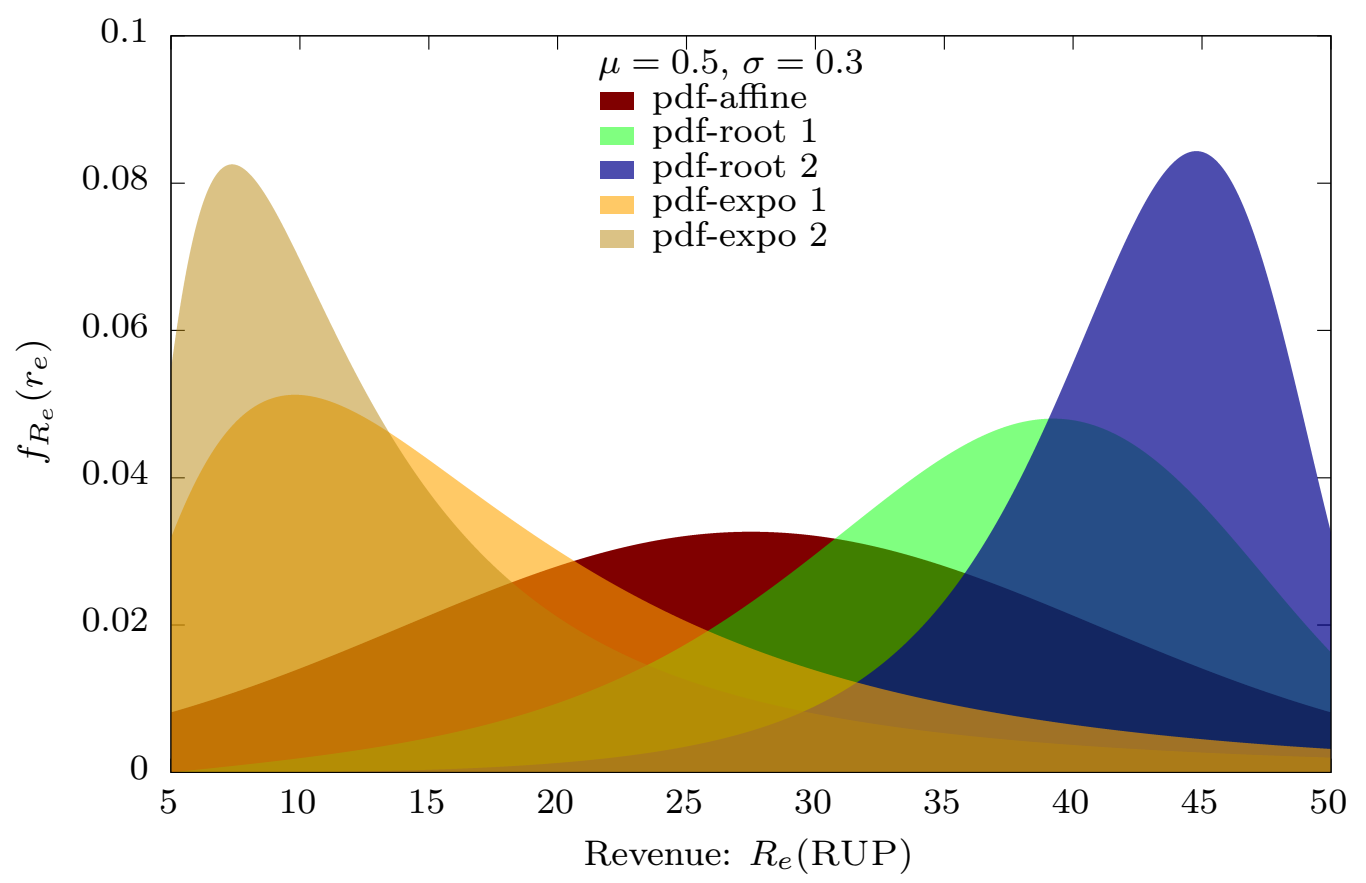

Figure 8: Different probability density functions of $R_{e}(\mathrm{RUP})$ : medium part quality

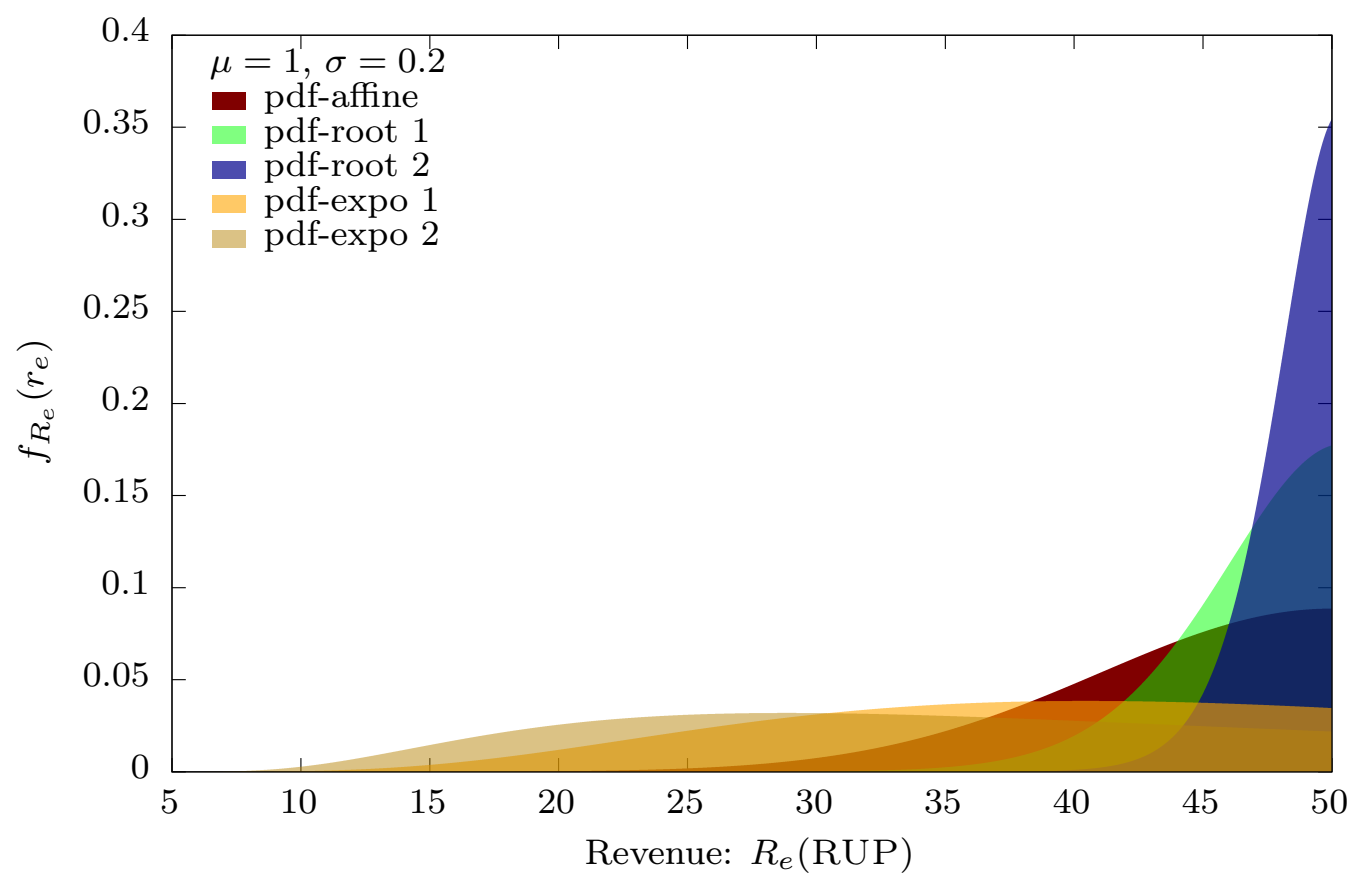

Figure 9: Different probability density functions of $R_{e}(\mathrm{RUP})$ : good part quality

In the next section, the optimization model is presented. The optimization will provide for each part of a product its optimal depth of disassembly according to the RUPs. 


\section{Optimization model and solution approach}

The objective is to maximize the profit of the disassembly process, which is defined as the difference between the net revenue of the recovered parts of the End-of-Life product and the cost of the corresponding disassembly tasks. To model the disassembly process planning problem, the following notations are introduced.

\subsection{Adopted notation}

$I$ : set of disassembly task indices: $I=\{1,2, \ldots, \mathrm{N}\}, \mathrm{N} \in \mathbb{N}^{*}$;

$L$ : set of all product part indices (subassemblies and components): $L=\{1,2, \ldots, \mathrm{L}\}, \mathrm{L} \in \mathbb{N}^{*}$;

$K$ : set of indices for the generated subassemblies: $K=\{0,1, \ldots, \mathrm{K}\}, \mathrm{K} \in \mathbb{N}, \mathrm{K}<\mathrm{L}$;

$A_{k}:$ a subassembly: $k \in K$

$B_{i}:$ a disassembly task: $i \in I$;

$L_{i}$ : set of indices of retrieved subassemblies and components by the execution of disassembly task $B_{i}, i \in I$;

$G_{\ell}:$ set of indices of tasks generating subassembly or component $\ell, \ell \in L$;

$D_{\ell}:$ set of indices of tasks disassembling subassembly $\ell, \ell \in L$;

$\widetilde{R_{e \ell}}$ : revenue generated by a subassembly or component $\ell, \ell \in L$ (see subsections 3.3 and 3.4), where $\widetilde{R_{e \ell}}$ is a function of $\widetilde{R U P}_{\ell}: \widetilde{R}_{e \ell}\left(\widetilde{\mathrm{RUP}}_{\ell}\right), \ell \in L ; \widetilde{\mathrm{RUP}}_{\ell}$ represents the remaining usage potential of a subassembly or component $\ell, \ell \in L$, as defined in subsection 3.2;

$c_{i}$ : cost of disassembly task $B_{i}, i \in I: c_{i}=c \cdot t_{i}, \forall i \in I ; t_{i}$ is the processing time of task $B_{i}$ and $c$ is a fixed cost per disassembly time unit, $i \in I$;

$P_{k}:$ set of indices of $A_{k}$ predecessors, $k \in K: P_{k}=\left\{i \mid B_{i}\right.$ precedes $\left.A_{k}\right\}$;

$S_{k}:$ set of indices of $A_{k}$ successors, $k \in K: S_{k}=\left\{i \mid A_{k}\right.$ precedes $\left.B_{i}\right\}$. 


\subsection{Decision variables}

Two kinds of variables are introduced. A variable of the first type consists to select or to not select a disassembly task of a disassembly process alternative. A variable of the second type is used as an intermediate in order to define the disassembly process revenue. These two types of variables are defined respectively as follows:

$x_{i}= \begin{cases}1, & \text { if disassembly task } B_{i}, i \in I \text { is selected; } \\ 0, & \text { otherwise. }\end{cases}$

$y_{\ell}= \begin{cases}0, & \text { if } \sum_{i \in G_{\ell}} x_{i}=1, \ell \in L \text { and } \sum_{i \in D_{\ell}} x_{i}=1(\ell \text { subassembly }) \\ 1, & \text { otherwise }\end{cases}$

Variable $y_{\ell}, \ell \in L$ means: for a subassembly with index $\ell \in L$, if a disassembly task with index $i, i \in G_{\ell}$ which generates $\ell$ is chosen and, next, another disassembly task $j, j \in D_{\ell}$, of the same disassembly process alternative, which disassembles it is also chosen, then its revenue $\widetilde{R_{e \ell}}$ is not taken into account while calculating the revenue of the whole disassembly process. Thus, only revenues of its components or subassemblies (not disassembled) are considered.

Note that $\sum_{i \in D_{\ell}} x_{i}$ and $\sum_{i \in G_{\ell}} x_{i} \in\{0,1\}, \forall \ell \in L$ ( $\ell$ subassembly) by the fact that only one disassembly task can be selected to generate the subassembly of index $\ell$ and only one disassembly task can be selected to disassemble it. It follows, then, that $y_{\ell}=1$ for any component (not subassembly) of index $\ell, \ell \in L$.

\subsection{Objective function and constraints}

The decision tool proposed in this study uses the optimization model defined hereafter. This model allows to determine a disassembly process alternative with the maximum profit while considering the quality or states of the subassemblies and components generated during the disassembly process. As mentioned in subsection 3.2, the state of a product (subassembly or component) is modeled using RUP. Hence, by taking the RUP of each subassembly and component, the optimization model allows to choose which components and subassemblies to retrieve in order to maximize 
the disassembly process profit. As explained in subsection 3.3, revenue $R_{e}$ of each component or subassembly depends on its RUP, or is a function of its RUP, i.e. $R_{e}(\mathrm{RUP})$.

The objective function is formulated as follows:

$\max \left\{\sum_{i \in I} \sum_{\ell \in L_{i}} \widetilde{R}_{e \ell} \cdot y_{\ell} \cdot x_{i}-\sum_{i \in I} c_{i} \cdot x_{i}\right\}$

The terms of the objective function represent, respectively, the earned profit of retrieved parts and the cost of the corresponding disassembly tasks.

The introduced constraints are described in the following. To start the selection of an optimal disassembly process, exactly one disassembly task, among all possible ones, must be chosen to disassemble the End-of-Life product (symbolized by $A_{0}$, see subsection 3.1). This is traduced by equation (2):

$$
\sum_{i \in S_{0}} x_{i}=1
$$

Constraints (3) formulate the precedence relationships among tasks and subassemblies and components. Note that, with this equation, a disassembly task could be not selected if the disassembly process to be retained as optimal is partial.

$$
\sum_{i \in S_{k}} x_{i} \leqslant \sum_{i \in P_{k}} x_{i}, \forall k \in K \backslash\{0\}
$$

Constraints (4) and (5), as previously mentioned, models an exclusion between the revenue of a subassembly (which is disassembled) and the revenue of its components. Constraints (5) are introduced in order to include in the retrained disassembly process revenues that are generated by selected components.

$$
\begin{aligned}
& \text { If } \sum_{i \in D_{\ell}} x_{i}=1 \text { and } \sum_{i \in G_{\ell}} x_{i}=1 \text { then } y_{\ell}=0, \forall \ell \in L(\ell \text { subassembly }) \\
& \left.y_{\ell}=1, \forall \ell \in L \text { ( } \ell \text { component }\right)
\end{aligned}
$$


Finally, the trivial constraints (6) define the possible values of the decision variables.

$x_{i}, y_{\ell} \in\{0,1\}, \forall i \in I, \forall \ell \in L$

The optimization model above in its actual form can not be solved using linear programming solvers. In fact, the total revenue term in the objective function (1) as well as constraints (4) are not linear. To linearize them, let $d_{\ell}=\sum_{i \in D_{\ell}} x_{i}, g_{\ell}=\sum_{i \in G_{\ell}} x_{i}, \forall \ell \in L$ ( $\ell$ subassembly) and $z_{i \ell}=$ $y_{\ell} \cdot x_{i}, \forall i \in I, \forall \ell \in L_{i}$. Then, the objective function becomes $\max \left\{\sum_{i \in I} \sum_{\ell \in L_{i}} \widetilde{R}_{e \ell} \cdot z_{i \ell}-\sum_{i \in I} c_{i} \cdot x_{i}\right\}$, such as $z_{i \ell} \leqslant y_{\ell}, z_{i \ell} \leqslant x_{i}$ and $z_{i \ell} \geqslant y_{\ell}+x_{i}-1$. Constraints (4) can then be replaced by $d_{\ell}+g_{\ell}-1 \leqslant h_{\ell}$ and $y_{\ell}=1-h_{\ell}, \forall \ell \in L\left(\ell\right.$ subassembly), where $h_{\ell} \in\{0,1\}$.

\subsection{Solution approach}

In order to solve the developed model, above, we consider different values of the revenue $\widetilde{R}_{e \ell}, \forall \ell \in L$, where $\ell$ represents a subassembly or a component. These values depend on: $\widehat{\mu}_{\ell}($ mean of $\widetilde{R_{e \ell}}$ ), $\widehat{\sigma}_{\ell}$ (standard deviation of $\widetilde{R}_{e \ell}$ ) and $\widehat{\bmod }_{\ell}$ (mode or most likely value of $\widetilde{R}_{e \ell}$ ), $\forall \ell \in L$.

Concretely, 6 values of $\widetilde{R}_{e \ell}, \forall \ell \in L$ will be considered: $R_{e \ell}=\widehat{\mu}_{\ell}, R_{e \ell}=\widehat{\bmod }_{\ell}, R_{e \ell}=\widehat{\mu}_{\ell} \pm \widehat{\sigma}_{\ell}$ and $R_{e \ell}=\widehat{\bmod }_{\ell} \pm \widehat{\sigma}_{\ell}, \forall \ell \in L$. The values of $\widehat{\mu}_{\ell}, \widehat{\bmod }_{\ell}$ and $\widehat{\sigma}_{\ell}$ of the revenue $\widetilde{R}_{e \ell}$ of each subassembly and component $\ell, \ell \in L$, are calculated using nonlinear optimizations with ALGLIB library and numerical integrations with a Gauss-Legendre quadrature rule.

Subsequently, the obtained problems will be solved using the IBM solver CPLEX 12.6.

\section{Numerical illustration: application to a remanufacturing industrial case}

The model (1)-(6) as well as the nonlinear optimizations and numerical integrations are implemented in Linux using $\mathrm{C}++$ on a PC with $8 \times \mathrm{CPU} 2.80 \mathrm{GHz}$ and 32 Go RAM. The model (1)-(6) is solved using ILOG CPLEX 12.6. It is applied to the ball point pen instance illustrated in Figure 1 and to a remanufacturing industrial case product: a Knorr-Bremse EBS 1 Channel Module, see Figure 10. This latter represents a real case study in the automotive part remanufacturing sector. Such a product is composed of at least 45 components; the corresponding and/or graph is given in Figure 11. Figure 12 offers a view of almost all components of the EBS 1 Channel Module. 


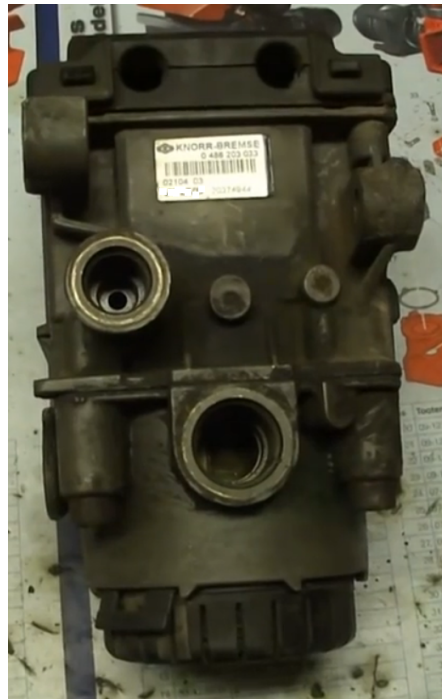

(a) Example of a returned product

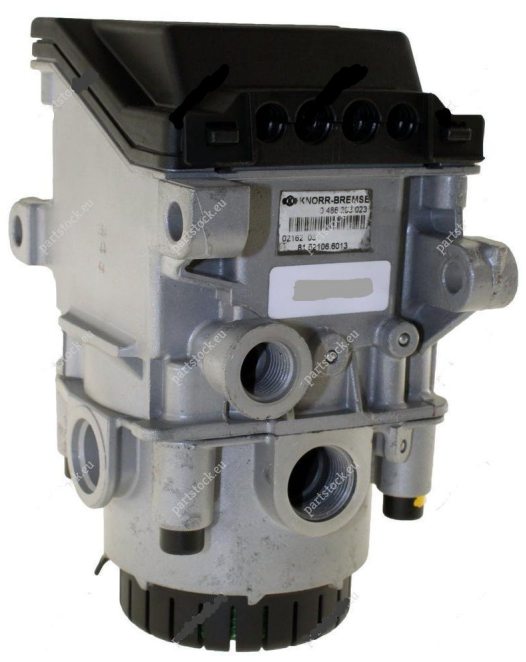

(b) Example of a remanufactured product

Figure 10: Remanufacturing industrial case product: Knorr-Bremse EBS 1 Channel Module

Table 2: Parameters of the two considered instances (Ball point pen and EBS 1 Channel Module)

\begin{tabular}{|l|ccccc|ccc|}
\cline { 6 - 8 } \multicolumn{1}{c|}{} & \multirow{2}{*}{} & N & \multirow{2}{*}{ K } & L & arcs & \multicolumn{3}{|c|}{ and-relations } \\
\cline { 6 - 9 } \multicolumn{1}{c|}{} & & & & & 0 & 1 & 2 \\
\hline Ball point pen & 20 & 13 & 23 & 41 & 5 & 9 & 6 \\
\hline EBS 1 CM & 72 & 35 & 67 & 175 & 6 & 29 & 37 \\
\hline
\end{tabular}

Table 3: The three part state categories according to the part quality: bad, medium and good

\begin{tabular}{|l|l|l|c|l|l|}
\hline state '0' & bad quality & & 0 & & 0.2 \\
\hline state '1' & medium quality & \multirow{n}{*}{$\mu$} & 0.5 & $\sigma$ & 0.3 \\
\cline { 1 - 1 } & & 1 & & 0.2 \\
\hline
\end{tabular}

The input data describing each instance is given in Table 2. Recall that (see subsection 4.1) N represents the number of all disassembly tasks, $\mathrm{K}$ the number of all subassemblies of a product and $\mathrm{L}$ the number of all parts of a product (components and subassemblies). Columns 'and-relations' report the number of disassembly tasks with no successor in subcolumn ' 0 ', with one and-type arc in subcolumn ' 1 ' and with two and-type arcs in subcolumn '2'. Column 'arcs' gives the total number of and-type and or-type arcs.

For each component or subassembly $\ell \in L$, RUP follows a normal distribution truncated in 0 and 1 with parameters $\left(\mu_{\ell}, \sigma_{\ell}\right), \ell \in L$; i.e. $\operatorname{RUP}_{\ell} \rightsquigarrow \mathcal{N}_{[0,1]}\left(\mu_{\ell}, \sigma_{\ell}\right), \ell \in L$. All components and subassemblies are classified into 3 categories corresponding to different levels of RUL: category '0' (bad state), category '1' (medium state) and category '2' (good state), see Section 3.4. 


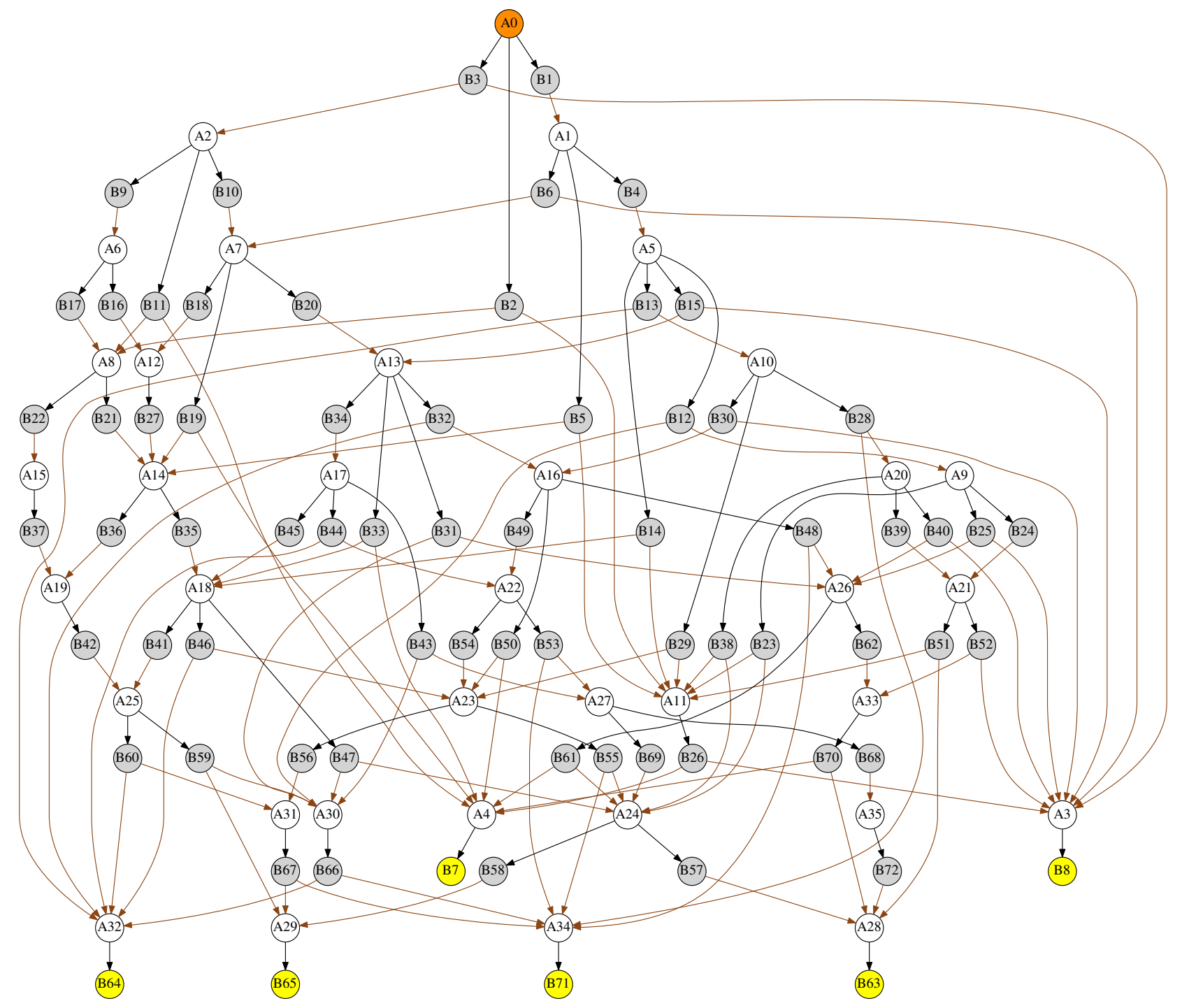

Figure 11: And/or graph of Knorr-Bremse EBS 1 Channel Module

The $\mu_{\ell}$ and $\sigma_{\ell}$ values, for a component or subassembly $\ell \in L$, are defined according to its state (or quality) category as shown in Table 3. These categories are assigned to all components and subassemblies of the two considered products according to a rule of common sense, see Table 4. For example, component 1 of the ball point pen (click button, Figure 1) being widely used during its operating period is classified in the category ' 0 '.

Parameters $a_{\ell}$ (minimum revenue) and $b_{\ell}$ (maximum revenue), for each component or subassembly $\ell \in L$, are defined as reported in Table 4. The upper bound corresponds to the resale price of an almost new used item. The value of the EBS 1 Channel Module as almost new used product is considered to be $796.6 €$, i.e. $b=796.6 €$; this value represents an estimate of its real 


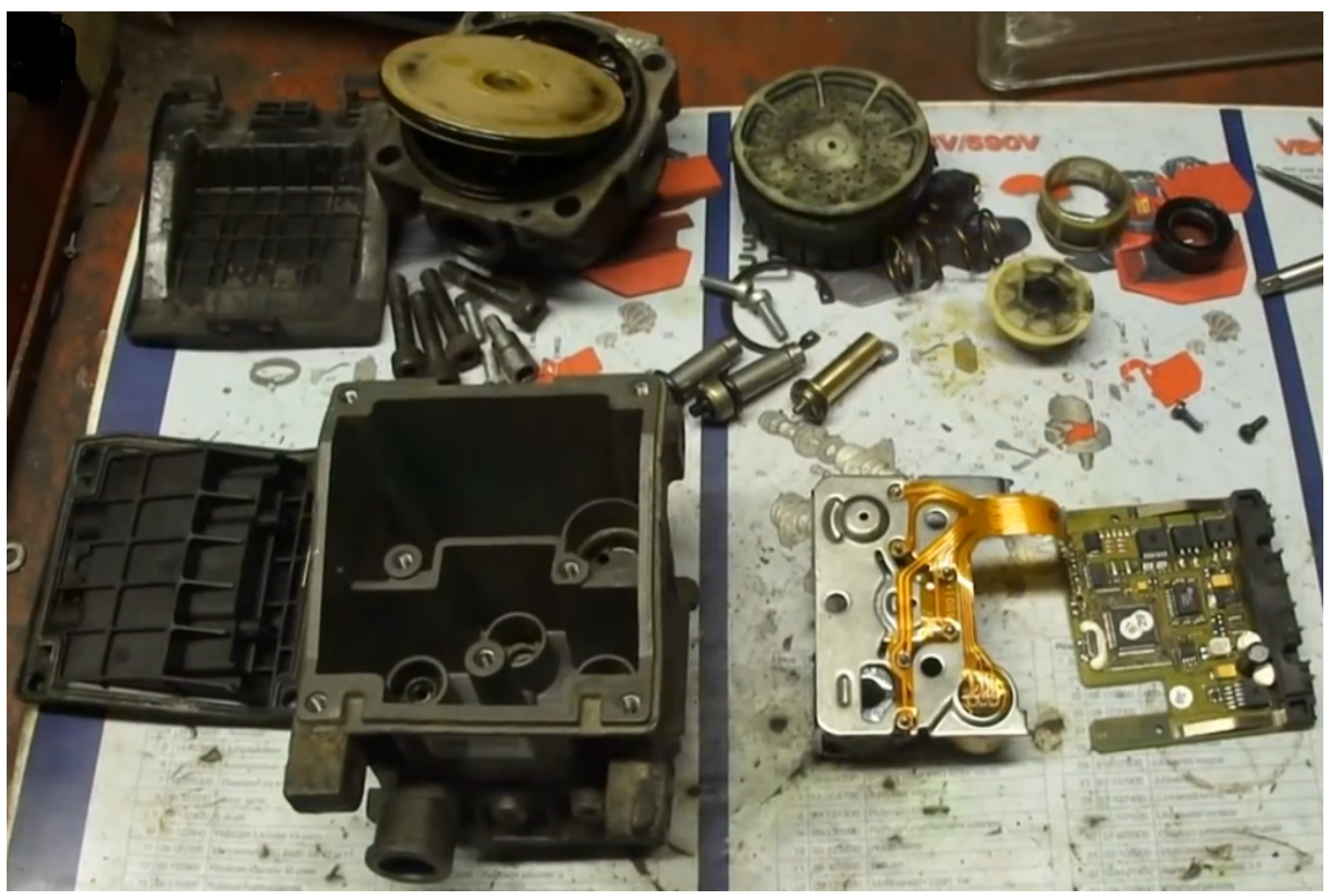

Figure 12: Components of the returned Knorr-Bremse EBS 1 Channel Module

average price in the market, see website 1 and website 2 . The resale value of a remanufactured EBS 1 Channel Module is around $100 €$, see website 3. The revenue which can be generated by recovering the material of this product is taken to be $1.49 €$. The latter is obtained by summing the different material values of all the product components. In order to get an approximation value for each material, prices of the corresponding raw materials on the market are taken into account. The values of $a, b$ of subassemblies are considered to be, resp., the sum of $a, b$ values of their components. This value, for $b$, is then corrected using a coefficient. Indeed, in the assembly process of EBS 1 Channel Module as in general, the value of assembled product is greater than the sum of its components values. In the case of EBS 1 Channel Module, this coefficient is taken equal to 5 . For the ball point pen, $b$ is taken equal to $7.5 €, a$ equal to 0.55 cents of $€$.

Costs of disassembly tasks are determined using data of job market in France. Indeed, for a fulltime job in an enterprise that applies the statutory weekly working time of 35 hours, an operator works approximatively 151.67 hours per month. The corresponding minimum salary per month is $1600 €$ (gross), all taxes included for the employer. Hence, the obtained cost of a disassembly task is 0.29 cents of $€$ per second. Disassembly task times, in seconds, are approximated by exploiting 
Table 4: State category and $a, b$ values for each product component or subassembly

\begin{tabular}{|c|c|c|c|c|c|c|c|c|c|c|c|c|c|c|c|c|c|c|c|}
\hline \multicolumn{16}{|c|}{ Knorr-Bremse EBS 1 Channel Module } & \multicolumn{4}{|c|}{ Ball point pen } \\
\hline 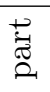 & $\frac{\pi}{i n}$ & $a$ & $b$ & 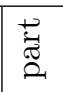 & 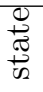 & $a$ & $b$ & 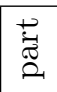 & $\Phi$ & $a$ & $b$ & $\begin{array}{l}\overrightarrow{\tilde{\tilde{\sigma}}} \\
\stackrel{2}{n}\end{array}$ & $\begin{array}{l}\underset{\Phi}{\Phi} \\
\underset{\omega}{\sigma}\end{array}$ & $a$ & $b$ & 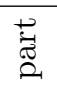 & 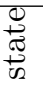 & $a$ & $b$ \\
\hline 1 & 2 & 0.28 & 1.0 & 24 & 0 & 0.40 & 100.0 & 47 & 1 & 148.96 & 78461.2 & 70 & 2 & 113.59 & 55406.2 & 1 & 0 & 0.003 & 15.0 \\
\hline 2 & 2 & 0.28 & 1.0 & 25 & 2 & 10.00 & 1500.0 & 48 & 1 & 0.70 & 1211.5 & 71 & 1 & 126.63 & 58195.8 & 2 & 0 & 0.002 & 10.0 \\
\hline 3 & 2 & 0.06 & 1.0 & 26 & 1 & 7.00 & 1000.0 & 49 & 1 & 24.52 & 17741.8 & 72 & 2 & 125.80 & 57338.8 & 3 & 1 & 0.05 & 60.0 \\
\hline 4 & 2 & 0.06 & 1.0 & 27 & 2 & 2.20 & 20.0 & 50 & 1 & 148.82 & 76694.1 & 73 & 1 & 70.80 & 28013.4 & 4 & 0 & 0.140 & 20.0 \\
\hline 5 & 2 & 0.06 & 1.0 & 28 & 1 & 24.50 & 3000.0 & 51 & 1 & 148.13 & 77604.2 & 74 & 2 & 92.10 & 38119.5 & 5 & 0 & 0.132 & 5.0 \\
\hline 6 & 2 & 0.06 & 1.0 & 29 & 1 & 70.00 & 4500.0 & 52 & 1 & 148.36 & 75506.2 & 75 & 2 & 21.31 & 17269.0 & 6 & 2 & 0.09 & 10.0 \\
\hline 7 & 2 & 0.06 & 1.0 & 30 & 2 & 1.00 & 300.0 & 53 & 1 & 115.23 & 59803.3 & 76 & 2 & 111.58 & 53325.9 & 7 & 1 & 0.066 & 5.0 \\
\hline 8 & 2 & 0.06 & 1.0 & 31 & 2 & 0.35 & 20.0 & 54 & 1 & 127.33 & 59407.3 & 77 & 2 & 1.45 & 2068.5 & 8 & 2 & 0.011 & 10.0 \\
\hline 9 & 2 & 0.06 & 1.0 & 32 & 1 & 0.60 & 500.0 & 55 & 1 & 146.81 & 74613.8 & 78 & 1 & 104.53 & 46671.3 & 9 & 1 & 0.033 & 100.0 \\
\hline 10 & 1 & 2.10 & 3.0 & 33 & 0 & 0. & & 56 & 1 & & & 79 & 2 & 19.3 & 15188.7 & 10 & 1 & 0.105 & 15.0 \\
\hline 11 & 1 & 2.10 & 3.0 & 34 & 0 & 0.10 & 30.0 & 57 & 1 & 147.53 & 74649.2 & 80 & 1 & 103.7 & 45814.3 & 11 & 1 & 0.411 & 379.5 \\
\hline 12 & 1 & 2.10 & 3.0 & 35 & 0 & 0.10 & 30.0 & 58 & 1 & 148.12 & 75482.5 & ${ }^{\circ}$ & $\cdot$ & 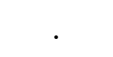 & . & 12 & 1 & 0.356 & 166.8 \\
\hline 13 & 1 & 2.10 & 3.0 & 36 & 0 & 0.10 & 30.0 & 59 & 1 & 114.53 & 56848.3 & $\cdot$ & 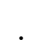 & . & . & 13 & 1 & 0.251 & 382.2 \\
\hline 14 & 2 & 0.02 & 2.0 & 37 & 0 & 0.10 & 30.0 & 60 & 2 & 114.43 & 58384.9 & 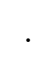 & . & 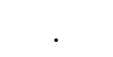 & . & & 0 & 0.195 & 284.8 \\
\hline 15 & 2 & 0.01 & 3.0 & 38 & 0 & 0.10 & 30.0 & 61 & 1 & 146.11 & 73402.2 & . & $\cdot$ & 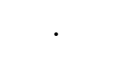 & . & 15 & 0 & 0.218 & 72.7 \\
\hline 16 & 0 & 0.10 & 50.0 & 39 & 0 & 0.10 & 30.0 & 62 & 1 & 147.29 & 74625.6 & 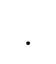 & . & 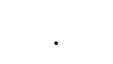 & . & 16 & 0 & 0.055 & 109.5 \\
\hline 17 & 0 & 0.10 & 50.0 & 40 & 0 & 0.10 & 30.0 & 63 & 1 & 114.39 & 56824.7 & . & . & 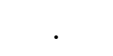 & . & 17 & 0 & 0.207 & 59.9 \\
\hline 18 & 0 & 0.50 & 10.0 & 41 & 0 & 0.10 & 30.0 & 64 & 2 & 113.83 & 55429.9 & $\cdot$ & . & 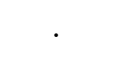 & . & 18 & 1 & 0.149 & 189.7 \\
\hline 19 & 1 & 4.20 & 500.0 & 42 & 0 & 0.10 & 30.0 & 65 & 1 & 127.33 & 59407.3 & 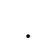 & . & 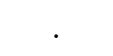 & . & 19 & 0 & 0.005 & 74.0 \\
\hline 20 & 1 & 4.20 & 500.0 & 43 & 0 & 0.10 & 50.0 & 66 & 1 & 105.23 & 47882.8 & . & . & . & 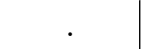 & 20 & 0 & 0.19 & 188.7 \\
\hline 21 & 1 & 13.20 & 800.0 & 44 & 0 & 0.10 & 50.0 & 67 & 1 & 145.28 & 72545.2 & $\cdot$ & . & 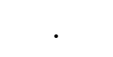 & . & 21 & 0 & 0.141 & 26.8 \\
\hline 22 & 1 & 0.20 & 75.0 & 45 & 0 & 0.10 & 30.0 & 68 & $t_{2}$ & 112.38 & 54744.3 & . & . & . & . & 22 & 1 & 0.044 & 149.2 \\
\hline 23 & 1 & 0.40 & 100.0 & 46 & 1 & 149.06 & 76717.7 & 69 & 2 & 92.90 & 39537.9 & & . & . & 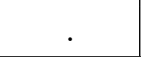 & 23 & 1 & 0.138 & 175.4 \\
\hline
\end{tabular}

some videos in which operators disassemble the products; for Knorr-Bremse EBS 1 Channel Module see website 4 .

Tables 5, 6, 7, 8, 9, and 10 gather the obtained optimization results for different values of $R_{e \ell}, \ell \in L$. For each value of $R_{e \ell}, \ell \in L$, six different cases are studied (see subsections 3.3 and $3.4)$ :

Affine $\quad$ each revenue $\widetilde{R_{e \ell}}, \ell \in L$ is a function of type (affine).

Root 1 each revenue $\widetilde{R}_{e \ell}, \ell \in L$ is a function of type (root 1 ).

Root 2 each revenue $\widetilde{R_{e \ell}}, \ell \in L$ is a function of type (root 2).

Expo 1 each revenue $\widetilde{R_{e \ell}}, \ell \in L$ is a function of type (expo 1$)$. 
Expo 2 each revenue $\widetilde{R}_{e \ell}, \ell \in L$ is a function of type (expo 2).

Mixture $20 \%$ of all revenues $\widetilde{R_{e \ell}}, \forall \ell \in L$ are of type (affine), $20 \%$ of type (root 1 ), $20 \%$ of type (root 2), $20 \%$ of type (expo 1) and $20 \%$ of type (expo 2). In this case, the function assigned to each component or subassembly is selected randomly.

Columns 'obj.', 'nbr-tasks', 'select-tasks', 'sub-assmbls.', 'components' and 'time(s)' indicate, respectively, the objective function value, the number of tasks of the selected disassembly alternative, the selected disassembly tasks (i.e. the disassembly level), the generated subassemblies, the generated components and the resolution time in seconds.

Results of Tables 5, 6, 7, 8, 9, and 10 can be summarized as follows: the profit of the disassembly process depends not only on the sequence and level of disassembly but also on the state or quality of the product. In fact, profit is the difference between revenues $\left(R_{e}\right)$ of the components and/or subassemblies and costs $c_{i}$ of the disassembly tasks. As disassembly costs are known and fixed, then the profit in our case depends mainly on the revenues of the components and/or subassemblies. Revenues are random and they are functions of states of the components and subassemblies.

Table 5: Optimization results of ball point pen and EBS 1 Channel Module: $R_{e \ell}=\widehat{\mu}_{\ell}, \forall \ell \in L$

\begin{tabular}{|c|c|c|c|c|c|c|c|}
\hline & obj. & nbr-tasks & select-tasks & sub-assmbls. & components & time $(\mathrm{s})$ & \\
\hline Affine & 243.5 & 9 & 26 & $3 ; 4$ & 10 & 1.09 & \\
\hline Root 1 & 374.6 & 9 & 26 & $3 ; 4$ & 10 & 1.07 & 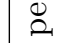 \\
\hline Root 2 & 491.2 & 9 & 26 & $3 ; 4$ & 10 & 1.14 & $\vec{\Xi}$ \\
\hline Expo 1 & 42.7 & 9 & 261017 & $3 ; 9$ & $3 ; 4 ; 10$ & 1.11 & 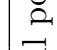 \\
\hline Expo 2 & 26.1 & 9 & 261017 & $3 ; 9$ & $3 ; 4 ; 10$ & 1.12 & $\overline{\mathscr{n}}$ \\
\hline Mixture & 415.5 & 9 & 2610 & $3 ; 9 ; 10$ & 10 & 1.09 & \\
\hline Affine & 63550.2 & 12 & 14153443 & $3 ; 27 ; 30$ & $\begin{array}{l}3 ; 4 ; 5 ; 6 ; 7 ; 8 ; 9 ; 14 ; 15 \\
16 ; 17 ; 18 ; 32 ; 33\end{array}$ & 2.9 & \\
\hline Root 1 & 69378.6 & 12 & 310203443 & $3 ; 27 ; 30$ & $\begin{array}{l}3 ; 4 ; 5 ; 6 ; 7 ; 8 ; 9 ; 14 ; 15 \\
16 ; 17 ; 18 ; 32 ; 33\end{array}$ & 3.5 & $\begin{array}{l}0 \\
\frac{0}{3} \\
0\end{array}$ \\
\hline Root 2 & 72710.7 & 12 & 310203443 & $3 ; 27 ; 30$ & $\begin{array}{l}3 ; 4 ; 5 ; 6 ; 7 ; 8 ; 9 ; 14 ; 15 \\
16 ; 17 ; 18 ; 32 ; 33\end{array}$ & 3.2 & $\underset{\Xi}{\stackrel{\Xi}{\Xi}}$ \\
\hline Expo 1 & 34540.7 & 12 & 14153443 & $3 ; 27 ; 30$ & $\begin{array}{l}3 ; 4 ; 5 ; 6 ; 7 ; 8 ; 9 ; 14 ; 15 \\
16 ; 17 ; 18 ; 32 ; 33\end{array}$ & 2.8 & $\underset{-1}{0}$ \\
\hline Expo 2 & 26689.4 & 12 & 16203443 & $3 ; 27 ; 30$ & $\begin{array}{l}3 ; 4 ; 5 ; 6 ; 7 ; 8 ; 9 ; 14 ; 15 \\
16 ; 17 ; 18 ; 32 ; 33\end{array}$ & 4.3 & 周 \\
\hline Mixture & 71287.1 & 12 & 222 & $11 ; 15$ & $\begin{array}{l}10 ; 11 ; 12 ; 13 ; 15 ; 16 ; 17 \\
18 ; 24 ; 33 ; 40 ; 41 ; 42 ; 43\end{array}$ & 3.3 & \\
\hline
\end{tabular}


Table 6: Optimization results of ball point pen and EBS 1 Channel Module: $R_{e \ell}=\widehat{\mu}_{\ell}-\widehat{\sigma}_{\ell}, \forall \ell \in L$

\begin{tabular}{|c|c|c|c|c|c|c|c|}
\hline & obj. & nbr-tasks & select-tasks & sub-assmbls. & components & time $(\mathrm{s})$ & \\
\hline Affine & 121.4 & 9 & 261017 & $3 ; 9$ & $3 ; 4 ; 10$ & 1.09 & \\
\hline Root 1 & 254.9 & 9 & 26 & $3 ; 4$ & 10 & 1.10 & 离 \\
\hline Root 2 & 401.1 & 9 & 26 & $3 ; 4$ & 10 & 1.09 &.$\ddot{\Xi}$ \\
\hline Expo 1 & 42.7 & 9 & 261017 & $3 ; 9$ & $3 ; 4 ; 10$ & 1.12 & 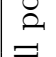 \\
\hline Expo 2 & 26.1 & 9 & 261017 & $3 ; 9$ & $3 ; 4 ; 10$ & 1.12 & ๑ี \\
\hline Mixture & 306.6 & 9 & 2610 & $3 ; 9 ; 10$ & 10 & 1.09 & \\
\hline Affine & 54146.7 & 12 & 310203443 & $3 ; 27 ; 30$ & $\begin{array}{l}3 ; 4 ; 5 ; 6 ; 7 ; 8 ; 9 ; 14 ; 15 \\
16 ; 17 ; 18 ; 32 ; 33\end{array}$ & 2.9 & \\
\hline Root 1 & 63884.0 & 12 & 310203443 & $3 ; 27 ; 30$ & $\begin{array}{l}3 ; 4 ; 5 ; 6 ; 7 ; 8 ; 9 ; 14 ; 15 \\
16 ; 17 ; 18 ; 32 ; 33\end{array}$ & 2.9 & $\frac{0}{\Xi}$ \\
\hline Root 2 & 69702.4 & 12 & 14153443 & $3 ; 27 ; 30$ & $\begin{array}{l}3 ; 4 ; 5 ; 6 ; 7 ; 8 ; 9 ; 14 ; 15 \\
16 ; 17 ; 18 ; 32 ; 33\end{array}$ & 2.9 & 竞 \\
\hline Expo 1 & 14852 & 12 & 16203443 & $3 ; 27 ; 30$ & $\begin{array}{l}3 ; 4 ; 5 ; 6 ; 7 ; 8 ; 9 ; 14 ; 15 \\
16 ; 17 ; 18 ; 32 ; 33\end{array}$ & 2.9 & 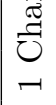 \\
\hline Expo 2 & 6782.7 & 12 & 14153443 & $3 ; 27 ; 30$ & $\begin{array}{l}3 ; 4 ; 5 ; 6 ; 7 ; 8 ; 9 ; 14 ; 15 \\
16 ; 17 ; 18 ; 32 ; 33\end{array}$ & 3.0 & 公 \\
\hline Mixture & 66677.6 & 12 & 222 & $11 ; 15$ & $\begin{array}{l}10 ; 11 ; 12 ; 13 ; 15 ; 16 ; 17 \\
18 ; 24 ; 33 ; 40 ; 41 ; 42 ; 43\end{array}$ & 3.9 & \\
\hline
\end{tabular}

Table 7: Optimization results of ball point pen and EBS 1 Channel Module: $R_{e \ell}=\widehat{\mu}_{\ell}+\widehat{\sigma}_{\ell}, \forall \ell \in L$

\begin{tabular}{|c|c|c|c|c|c|c|c|}
\hline & obj. & nbr-tasks & select-tasks & sub-assmbls. & components & time(s) & \\
\hline Affine & 372.6 & 9 & 26 & $3 ; 4$ & 10 & 1.08 & \\
\hline Root 1 & 494.3 & 9 & 26 & $3 ; 4$ & 10 & 1.27 & $\bar{\Xi}$ \\
\hline Root 2 & 581.4 & 9 & 26 & $3 ; 4$ & 10 & 1.11 &.$\vec{\Xi}$ \\
\hline Expo 1 & 119.4 & 9 & 261017 & $3 ; 9$ & $3 ; 4 ; 10$ & 1.08 & 2 \\
\hline Expo 2 & 88.4 & 9 & 261017 & $3 ; 9$ & $3 ; 4 ; 10$ & 1.10 & అี \\
\hline Mixture & 526.8 & 9 & 2610 & $3 ; 9 ; 10$ & 10 & 1.12 & \\
\hline Affine & 72953.7 & 12 & 310203443 & $3 ; 27 ; 30$ & $\begin{array}{l}3 ; 4 ; 5 ; 6 ; 7 ; 8 ; 9 ; 14 ; 15 \\
16 ; 17 ; 18 ; 32 ; 33\end{array}$ & 3.0 & \\
\hline Root 1 & 74873.2 & 12 & 16203443 & $3 ; 27 ; 30$ & $\begin{array}{l}3 ; 4 ; 5 ; 6 ; 7 ; 8 ; 9 ; 14 ; 15 \\
16 ; 17 ; 18 ; 32 ; 33\end{array}$ & 3.0 & $\frac{0}{\Xi}$ \\
\hline Root 2 & 76084.2 & 12 & 222 & $11 ; 15$ & $\begin{array}{l}10 ; 11 ; 12 ; 13 ; 15 ; 16 ; 17 \\
18 ; 24 ; 33 ; 40 ; 41 ; 42 ; 43\end{array}$ & 3.0 & $\begin{array}{l}\Sigma \\
\overrightarrow{0} \\
\Xi\end{array}$ \\
\hline Expo 1 & 54515.9 & 12 & 310203443 & $3 ; 27 ; 30$ & $\begin{array}{l}3 ; 4 ; 5 ; 6 ; 7 ; 8 ; 9 ; 14 ; 15 \\
16 ; 17 ; 18 ; 32 ; 33\end{array}$ & 2.9 & 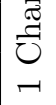 \\
\hline Expo 2 & 46828.7 & 12 & 310203443 & $3 ; 27 ; 30$ & $\begin{array}{l}3 ; 4 ; 5 ; 6 ; 7 ; 8 ; 9 ; 14 ; 15 \\
16 ; 17 ; 18 ; 32 ; 33\end{array}$ & 2.9 & 粪 \\
\hline Mixture & 75898.3 & 12 & 222 & $11 ; 15$ & $\begin{array}{l}10 ; 11 ; 12 ; 13 ; 15 ; 16 ; 17 \\
18 ; 24 ; 33 ; 40 ; 41 ; 42 ; 43\end{array}$ & 3.0 & \\
\hline
\end{tabular}

Thus, the profit of the disassembly process depends on the sequence and level of disassembly of the product. The level of disassembly is itself dependent on the states (quality) of the components 
and subassemblies.

Figures 13 and 14 show in detail the sequence (alternative) and the level of disassembly returned for each revenue function type of components and subassemblies. Figure 13 illustrates the ball point

Table 8: Optimization results of ball point pen and EBS 1 Channel Module: $R_{e \ell}=\widehat{\bmod }_{\ell}, \forall \ell \in L$

\begin{tabular}{|c|c|c|c|c|c|c|c|}
\hline & obj. & nbr-tasks & select-tasks & sub-assmbls. & components & time $(\mathrm{s})$ & \\
\hline Affine & 226.8 & 9 & 261017 & $3 ; 9$ & $3 ; 4 ; 10$ & 1.09 & \\
\hline Root 1 & 408.4 & 9 & 26 & $3 ; 4$ & 10 & 1.09 & $\stackrel{D}{2}$ \\
\hline Root 2 & 534.1 & 9 & 26 & $3 ; 4$ & 10 & 1.12 &. \\
\hline Expo 1 & 0.324 & 9 & 25111318 & $4 ; 13$ & $5 ; 6 ; 7 ; 8$ & 1.10 & \\
\hline Expo 2 & 0.327 & 9 & 1 & 1 & 4 & 1.11 & ๑ే \\
\hline Mixture & 458.6 & 9 & 2610 & $3 ; 9 ; 10$ & 10 & 1.13 & \\
\hline Affine & 75412.0 & 12 & 14153443 & $3 ; 27 ; 30$ & $\begin{array}{l}3 ; 4 ; 5 ; 6 ; 7 ; 8 ; 9 ; 14 ; 15 \\
16 ; 17 ; 18 ; 32 ; 33\end{array}$ & 2.9 & \\
\hline Root 1 & 75909.3 & 12 & 14153443 & $3 ; 27 ; 30$ & $\begin{array}{l}3 ; 4 ; 5 ; 6 ; 7 ; 8 ; 9 ; 14 ; 15 \\
16 ; 17 ; 18 ; 32 ; 33\end{array}$ & 2.9 & \\
\hline Root 2 & 76158.4 & 12 & 16203443 & $3 ; 27 ; 30$ & $\begin{array}{l}3 ; 4 ; 5 ; 6 ; 7 ; 8 ; 9 ; 14 ; 15 \\
16 ; 17 ; 18 ; 32 ; 33\end{array}$ & 2.9 & \\
\hline Expo 1 & 15616.1 & 12 & 14153443 & $3 ; 27 ; 30$ & $\begin{array}{l}3 ; 4 ; 5 ; 6 ; 7 ; 8 ; 9 ; 14 ; 15 \\
16 ; 17 ; 18 ; 32 ; 33\end{array}$ & 2.8 & en \\
\hline Expo 2 & 39298.5 & 12 & 3 & $2 ; 3$ & . & 2.7 & $\frac{\rho}{|c|}$ \\
\hline Mixture & 71287.1 & 12 & 310203443 & $3 ; 27 ; 30$ & $\begin{array}{l}3 ; 4 ; 5 ; 6 ; 7 ; 8 ; 9 ; 14 ; 15 ; \\
16 ; 17 ; 18 ; 32 ; 33\end{array}$ & 3.3 & \\
\hline
\end{tabular}

Table 9: Optimization results of ball point pen and EBS 1 Channel Module: $R_{e \ell}=\widehat{\bmod }_{\ell}-\widehat{\sigma}_{\ell}, \forall \ell \in L$

\begin{tabular}{|c|c|c|c|c|c|c|c|}
\hline & obj. & nbr-tasks & select-tasks & sub-assmbls. & components & time(s) & \\
\hline Affine & 117.7 & 9 & 261017 & $3 ; 9$ & $3 ; 4 ; 10$ & 1.12 & \\
\hline Root 1 & 288.7 & 9 & 26 & $3 ; 4$ & 10 & 1.11 & Dे \\
\hline Root 2 & 444.0 & 9 & 26 & $3 ; 4$ & 10 & 1.10 &. \\
\hline Expo 1 & 0.324 & 9 & 25111318 & $4 ; 13$ & $6 ; 6 ; 7 ; 8$ & 1.09 & 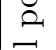 \\
\hline Expo 2 & 0.327 & 9 & 1 & 1 & 4 & 1.10 & صే \\
\hline Mixture & 349.7 & 9 & 2610 & $3 ; 9 ; 10$ & 10 & 1.11 & \\
\hline Affine & 66025.3 & 12 & 310203443 & $3 ; 27 ; 30$ & $\begin{array}{l}3 ; 4 ; 5 ; 6 ; 7 ; 8 ; 9 ; 14 ; 15 \\
16 ; 17 ; 18 ; 32 ; 33\end{array}$ & 3.0 & \\
\hline Root 1 & 70414.7 & 12 & 310203443 & $3 ; 27 ; 30$ & $\begin{array}{l}3 ; 4 ; 5 ; 6 ; 7 ; 8 ; 9 ; 14 ; 15 \\
16 ; 17 ; 18 ; 32 ; 33\end{array}$ & 2.9 & 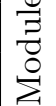 \\
\hline Root 2 & 73150.1 & 12 & 14153443 & $3 ; 27 ; 30$ & $\begin{array}{l}3 ; 4 ; 5 ; 6 ; 7 ; 8 ; 9 ; 14 ; 15 \\
16 ; 17 ; 18 ; 32 ; 33\end{array}$ & 3.0 & $\vec{\Xi}$ \\
\hline Expo 1 & 15613.5 & 12 & 14153443 & $3 ; 27 ; 30$ & $\begin{array}{l}3 ; 4 ; 5 ; 6 ; 7 ; 8 ; 9 ; 14 ; 15 \\
16 ; 17 ; 18 ; 32 ; 33\end{array}$ & 2.8 & $\begin{array}{l}\vec{D} \\
-\overrightarrow{0} \\
\text { की }\end{array}$ \\
\hline Expo 2 & 27926.7 & 12 & 3 & $2 ; 3$ & . & 2.8 & 公 \\
\hline Mixture & 70573.9 & 12 & 222 & $11 ; 15$ & $\begin{array}{l}10 ; 11 ; 12 ; 13 ; 15 ; 16 ; 17 ; \\
18 ; 24 ; 33 ; 40 ; 41 ; 42 ; 43\end{array}$ & 2.9 & \\
\hline
\end{tabular}


pen case and Figure 14 illustrates the case of EBS 1 Channel Module. In order to identify easily the disassembly alternatives in Figures 13, 14 and to better understand the results in Tables 5, $6,7,8,9,10$, one different color is assigned to each alternative as shown in Table 11 . Table 12 illustrates the obtained disassembly alternative with the corresponding objective value for each type of revenue function and each value of this revenue $\left(R_{e \ell}, \forall \ell \in L\right)$.

We can see that the disassembly sequence corresponding to the maximum profit for both studied products depends on the revenue functions, see Figures 13, 14 and Table 12. It can be observed

Table 10: Optimization results of ball point pen and EBS 1 Channel Module: $R_{e \ell}=\widehat{\bmod }_{\ell}+\widehat{\sigma}_{\ell}, \forall \ell \in L$

\begin{tabular}{|c|c|c|c|c|c|c|c|}
\hline & obj. & nbr-tasks & select-tasks & sub-assmbls. & components & time $(\mathrm{s})$ & \\
\hline Affine & 347.2 & 9 & 261017 & $3 ; 9$ & $3 ; 4 ; 10$ & 1.11 & \\
\hline Root 1 & 528.0 & 9 & 26 & $3 ; 4$ & 10 & 1.2 & 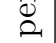 \\
\hline Root 2 & 573.6 & 9 & 26 & $3 ; 4$ & 10 & 1.3 &.$\vec{\Xi}$ \\
\hline Expo 1 & 75.1 & 9 & 261017 & $3 ; 9$ & $3 ; 4 ; 10$ & 1.12 & 2 \\
\hline Expo 2 & 60.7 & 9 & 261017 & $3 ; 9$ & $3 ; 4 ; 10$ & 1.13 & $\tilde{\tilde{\sigma}}$ \\
\hline Mixture & 569.9 & 9 & 2610 & $3 ; 9 ; 10$ & 10 & 1.08 & \\
\hline Affine & 75837.1 & 12 & 14153443 & $3 ; 27 ; 30$ & $\begin{array}{l}3 ; 4 ; 5 ; 6 ; 7 ; 8 ; 9 ; 14 ; 15 \\
16 ; 17 ; 18 ; 32 ; 33\end{array}$ & 3.0 & \\
\hline Root 1 & 76574.5 & 12 & 222 & $11 ; 15$ & $\begin{array}{l}10 ; 11 ; 12 ; 13 ; 15 ; 16 ; 17 ; \\
18 ; 24 ; 33 ; 40 ; 41 ; 42 ; 43\end{array}$ & 3.0 & 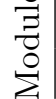 \\
\hline Root 2 & 76177.8 & 12 & 16203443 & $3 ; 27 ; 30$ & $\begin{array}{l}3 ; 4 ; 5 ; 6 ; 7 ; 8 ; 9 ; 14 ; 15 \\
16 ; 17 ; 18 ; 32 ; 33\end{array}$ & 2.9 & 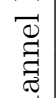 \\
\hline Expo 1 & 35591.2 & 12 & 14153443 & $3 ; 27 ; 30$ & $\begin{array}{l}3 ; 4 ; 5 ; 6 ; 7 ; 8 ; 9 ; 14 ; 15 \\
16 ; 17 ; 18 ; 32 ; 33\end{array}$ & 2.9 & $\begin{array}{l}\overrightarrow{0} \\
\vec{n} \\
\tilde{\omega}\end{array}$ \\
\hline Expo 2 & 50831.9 & 12 & 3 & $2 ; 3$ & . & 2.7 & $\stackrel{p}{q}$ \\
\hline Mixture & 75791.5 & 12 & 14153443 & $3 ; 27 ; 30$ & $\begin{array}{l}3 ; 4 ; 5 ; 6 ; 7 ; 8 ; 9 ; 14 ; 15 \\
16 ; 17 ; 18 ; 32 ; 33\end{array}$ & 2.9 & \\
\hline
\end{tabular}

Table 11: Colors representing all obtained disassembly alternatives for each instance

\begin{tabular}{|c|c|c|}
\hline Disassembly alternative & Alternative color & \\
\hline$B_{1}$ & & चี \\
\hline$B_{2} B_{6}$ & & $\vec{z}$ \\
\hline$B_{2} B_{6} B_{10}$ & & 要 \\
\hline$B_{2} B_{6} B_{10} B_{17}$ & & $\overline{\widetilde{乛}}$ \\
\hline $\begin{array}{lllll}B_{2} & B_{5} & B_{11} & B_{13} & B_{18} \\
\end{array}$ & & $\infty$ \\
\hline$B_{3}$ & & $\frac{0}{\Xi}$ \\
\hline$B_{2} B_{22}$ & & 苟 \\
\hline $\begin{array}{lllll}B_{1} & B_{4} & B_{15} & B_{34} & B_{43} \\
\end{array}$ & & z \\
\hline $\begin{array}{lllll}B_{3} & B_{10} & B_{20} & B_{34} & B_{43}\end{array}$ & & m \\
\hline $\begin{array}{lllll}B_{1} & B_{6} & B_{20} & B_{34} & B_{43}\end{array}$ & & 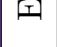 \\
\hline
\end{tabular}


that the level of disassembly for the same alternative depends on the type of revenue functions.

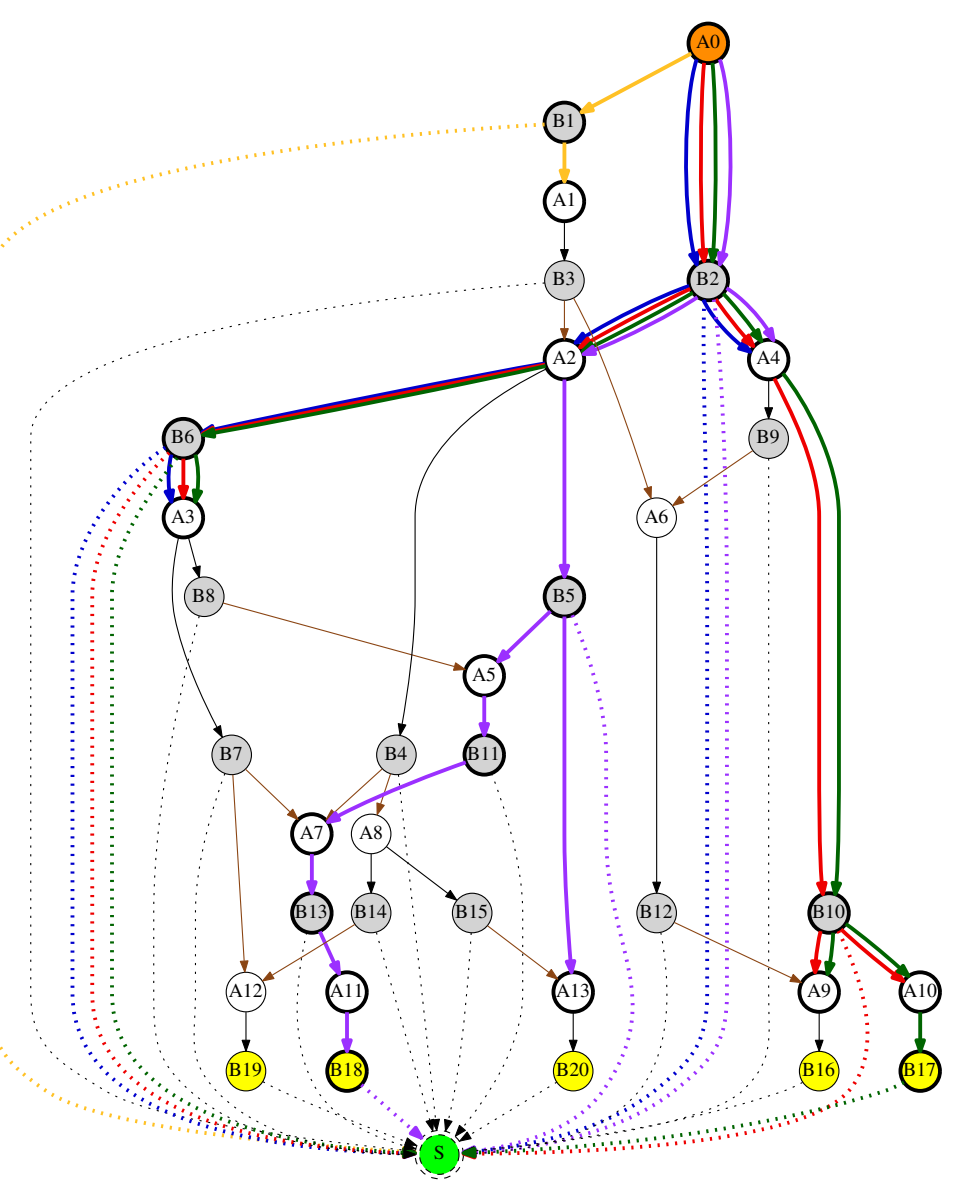

Figure 13: Alternatives and disassembly levels returned according to the type of revenue functions: Ball point pen

Table 12: Obtained disassembly alternative with the corresponding objective value for each revenue function type and each revenue value

\begin{tabular}{|c|c|c|c|c|c|c|c|}
\hline & $R_{e \ell}=\widehat{\mu}_{\ell}$ & $R_{e \ell}=\widehat{\mu}_{\ell}-\widehat{\sigma}_{\ell}$ & $R_{e \ell}=\widehat{\mu}_{\ell}+\widehat{\sigma}_{\ell}$ & $R_{e \ell}=\widehat{\bmod }_{\ell}$ & $R_{e \ell}=\widehat{\bmod }_{\ell}-\widehat{\sigma}_{\ell}$ & $R_{e \ell}=\widehat{\bmod }_{\ell}+\widehat{\sigma}_{\ell}$ & \\
\hline Affine & 243.5 & 121.4 & 372.6 & 226.8 & 117.7 & 347.2 & \\
\hline Root 1 & 374.6 & 254.9 & 494.3 & 408.4 & 288.7 & 528.0 & $\vec{\nabla}$ \\
\hline Root 2 & 491.2 & 401.1 & 581.4 & 534.1 & 444.0 & 573.6 &. \\
\hline Expo 1 & 42.7 & 42.7 & 119.4 & 0.324 & 0.324 & 75.1 & \\
\hline Expo 2 & 26.1 & 26.1 & 88.4 & 0.327 & 0.327 & 60.7 & n \\
\hline Mixture & 415.5 & 306.6 & 526.8 & 458.6 & 349.7 & 569.9 & \\
\hline Affine & 63550.2 & 54146.7 & 72953.7 & 75412.0 & 66025.3 & 75837.1 & \\
\hline Root 1 & 69378.6 & 63884.0 & 74873.2 & 75909.3 & 70414.7 & 76574.5 & $\bar{T}$ \\
\hline Root 2 & 72710.7 & 69702.4 & 76084.2 & 76158.4 & 73150.1 & 76177.8 & $\Sigma$ \\
\hline Expo 1 & 34540.7 & 14852 & 54515.9 & 15616.1 & 15613.5 & 35591.2 & $\frac{1}{2}$ \\
\hline Expo 2 & 26689.4 & 6782.7 & 46828.7 & 39298.5 & 27926.7 & 50831.9 & $\vec{I}$ \\
\hline Mixture & 71287.1 & 66677.6 & 75898.3 & 71287.1 & 70573.9 & 75791.5 & \\
\hline
\end{tabular}


For example, in the case of EBS 1 Channel Module, tasks $B_{3}$ and $B_{3} B_{10} B_{20} B_{34} B_{43}$ represent two different disassembly levels of the same disassembly alternative, see Figure 14. For the ball point pen case, tasks $B_{2} B_{6}, B_{2} B_{6} B_{10}$ and $B_{2} B_{6} B_{10} B_{17}$ represent three different disassembly levels of the same disassembly alternative, see Figure 13.

The results show also that for the same alternative and the same level of disassembly, values of the corresponding profits depend on the type of the revenue functions considered. As example, for EBS Channel Module case where $R_{e \ell}=\widehat{\bmod }_{\ell}, \forall \ell \in L$, functions Affine, Root1 and Expo1 define the same alternative $B_{1} B_{4} B_{15} B_{34} B_{43}$, but values of the objective functions are all different, see Table 8 and Table 12. For ball point pen case where $R_{e \ell}=\widehat{\mu}_{\ell}, \forall \ell \in L$, functions Affine, Root1 and Root2 define the same alternative $B_{2} B_{6}$ with different values of the objective functions, see

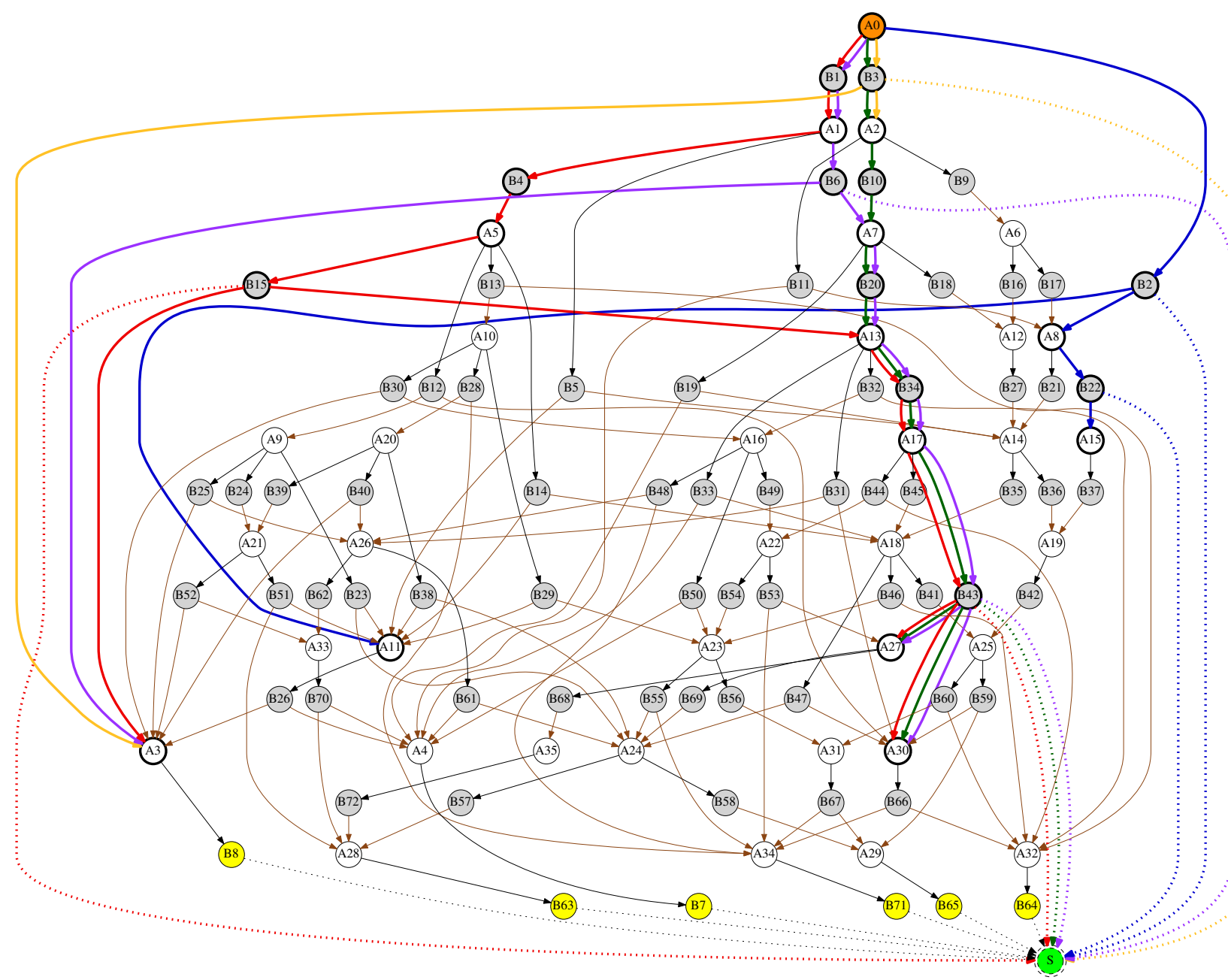

Figure 14: Alternatives and disassembly levels returned according to the type of revenue functions: EBS 1 Module 
Table 5 and Table 12. These values, for both products, are relatively important for functions of type (root 1) and (root 2), relatively low for functions of type (expo 1) and (expo 2). They are rather average for functions of type (affine).

In subsection 3.3, it is mentioned that the revenue $R_{e}$, which is a function of RUP, is linked to $a$ and $b$ values since $R_{e}(\mathrm{RUP}=1)=b$ and $R_{e}(\mathrm{RUP}=0)=a$, with $b>a>0$. From this relation, it is straightforward to deduce that the higher are $a$ and $b$ values the higher is the revenue $R_{e}$, and hence higher is the probability that the corresponding disassembly item will be selected for resale. As a consequence, the optimization results are also sensitive to estimation errors on the values of $a$ and $b$. To illustrate this fact, additional experiments are conducted using the ball point pen example. The obtained results are gothered in Table 13. The considered $a$ and $b$ values correspond to the original values, presented in Table 4 , and $\pm 20 \%$ of the original values; the revenue $R_{e \ell}$ value is taken to be equal to $\widehat{\mu}_{\ell}, \forall \ell \in L$, see Table 13 .

The results show that the returned disassembly alternative doesn't change if estimation errors on $a$ and $b$ values are of the same magnitute and sign for all the disassemby items. In fact, all the disassembly alternatives returned for $(a, b)-20 \%$ and $(a, b)+20 \%$ are exactly the same as

Table 13: Optimization results of the ball point pen with estimation errors on $a$ and $b$ values: $R_{e \ell}=\widehat{\mu}_{\ell}, \forall \ell \in L$

\begin{tabular}{|c|c|c|c|c|c|c|c|}
\hline & obj. & nbr-tasks & select-tasks & sub-assmbls. & components & time $(\mathrm{s})$ & \\
\hline Affine & 194.6 & 9 & 26 & $3 ; 4$ & 10 & 0.85 & \multirow{6}{*}{$\begin{array}{l}0 \\
0 \\
\text { o } \\
1 \\
1 \\
0 \\
0 \\
0\end{array}$} \\
\hline Root 1 & 299.5 & 9 & 26 & $3 ; 4$ & 10 & 0.75 & \\
\hline Root 2 & 392.8 & 9 & 26 & $3 ; 4$ & 10 & 0.75 & \\
\hline Expo 1 & 33.7 & 9 & 261017 & $3 ; 9$ & $3 ; 4 ; 10$ & 0.73 & \\
\hline Expo 2 & 20.4 & 9 & 261017 & $3 ; 9$ & $3 ; 4 ; 10$ & 0.77 & \\
\hline Mixture & 332.1 & 9 & 2610 & $3 ; 9 ; 10$ & 10 & 0.77 & \\
\hline Affine & 292.4 & 9 & 26 & $3 ; 4$ & 10 & 0.85 & \multirow{6}{*}{$\begin{array}{l}\Delta 0 \\
\text { o } \\
+ \\
+ \\
0 \\
0 \\
0\end{array}$} \\
\hline Root 1 & 449.8 & 9 & 26 & $3 ; 4$ & 10 & 0.78 & \\
\hline Root 2 & 589.7 & 9 & 26 & $3 ; 4$ & 10 & 0.77 & \\
\hline Expo 1 & 51.7 & 9 & 261017 & $3 ; 9$ & $3 ; 4 ; 10$ & 0.76 & \\
\hline Expo 2 & 31.7 & 9 & 261017 & $3 ; 9$ & $3 ; 4 ; 10$ & 0.78 & \\
\hline Mixture & 499.0 & 9 & 2610 & $3 ; 9 ; 10$ & 10 & 0.77 & \\
\hline Affine & 249.9 & 9 & 2661017 & $3 ; 9$ & $3 ; 4 ; 10$ & 0.90 & \multirow{6}{*}{ 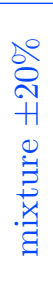 } \\
\hline Root 1 & 381.7 & 9 & 2610 & $3 ; 9 ; 10$ & 10 & 0.76 & \\
\hline Root 2 & 502.2 & 9 & 2610 & $3 ; 9 ; 10$ & 10 & 0.77 & \\
\hline Expo 1 & 44.4 & 9 & 261017 & $3 ; 9$ & $3 ; 4 ; 10$ & 0.76 & \\
\hline Expo 2 & 27.2 & 9 & 261017 & $3 ; 9$ & $3 ; 4 ; 10$ & 0.77 & \\
\hline Mixture & 438.1 & 9 & 2610 & $3 ; 9 ; 10$ & 10 & 0.76 & \\
\hline
\end{tabular}


the ones returned for original values of $a$ and $b$ (compare with Table 5). However, the objective values for the same alternatives in the case of original $a$ and $b$ values are greater than the ones of $(a, b)-20 \%$ and smaller than the ones of $(a, b)+20 \%$.

The results show also, as expected, that the optimal disassembly alternative depends on the estimated values of parameters $a$ and $b$ when estimation errors are of different sign, even if they are of same magnitude. Indeed, in the case of original $a$ and $b$ values (Table 5 ), the disassembly alternative returned for functions of types (affine), (root $\mathbf{1})$ and (root 2 ) is $B_{2} B_{6}$. But, in the case of mixture $\pm 20 \%$, the alternative $B_{2} B_{6} B_{10} B_{17}$ is returned for function of type (affine) while alternative $B_{2} B_{6} B_{10}$ is returned for functions of types (root $\mathbf{1}$ ) and (root 2).

The results of this section show the applicability of the developed optimization model and solution approach in real disassembly context. Indeed, the computational time is short enough to give to the decision maker the opportunity to generate different disassembly alternatives depending on the profit expected from the retrieved parts. The profit itself depends on the quality of the products. This model helps to make a decision on the disassembly alternative to be retained as disassembly process. Therefore, the choice between complete or partial disassembly can be made on the basis of the economic arguments.

When considering industrial issues the results allow setting the level of disassembly in order to design the disassembly process. Therefore, the choice between complete or partial disassembly can be made on the basis of the economic arguments, as cited just above. Nevertheless, since the parts are reused and reassembled, some additional cost might be considered such as, for instance, warranty and re-certification costs. In the pen example, such costs are insignificant while for the EBS module they are supported by the customer of the disassembled parts. Indeed, such customer, since it reassembles the EBS module, should re-certificate it for selling to car repair shop. As an example, Indra could ask a subcontractor to disassemble such used modules while performing the reassembling and re-certifying the refurbished ones.

The sensibility of results to product quality, i.e. the RUP, highlights 2 key points. First, the assessment of the product quality is mandatory for industrial issues. Not just once but periodically in order to re-optimize the disassembly level if it changes and, then, re-organize the disassembly 
process if required. Second, such assessment must be reliable and accurate. This requirement ensures the confidence in the results and thus in the industrial decision to be made.

In link with subsection 3.2 , in the case of the pen, only the $3^{\text {rd }}$ method applies: inspection of a sample of pens can be performed to statistically evaluate the RUPs of its parts. Since failure issues for the pen are not critical, visual inspection could be sufficient. For the EBS module, however, no monitoring of the module is available, only mileage of the truck might be known. In such case, the $1^{\text {st }}$ method does not apply. Based on the return of experience, from truck repairer,

weak parts are identified and reliability functions could be associated with them leading to RUP evaluation based on the $2^{\text {nd }}$ method. The $3^{\text {rd }}$ method should be applied to other parts like in the pen case. Nevertheless, some deeper inspection might be required for specific parts involved in security issues. Hence, destructive and non-destructive tests could be used. Generally speaking, the results of these RUP evaluations lead to build histograms. From such histograms, the most suitable PDFs can be identified. For instance, a normality test can be conducted. Based on the obtained results, $\mu_{l}$ and $\sigma_{l}$ are identified, not limited only to the 3 pairs of values given in the 2 examples. Other distributions, hence, can be considered.

Sensitivity of the results to the revenue leads, for industrial decision maker, to follow the resale price of the parts and raw materials. Disassembly level optimization should be redone when resale price evolves. The evolution of resale price is mainly market driven. Indeed, some market intelligence would allow anticipating resale price.

Finally, since the computation time is quite low, around 3s for the EBS module, several scenarios could be evaluated to make the best decision.

\section{Conclusion}

The disassembly process plays a key role in revalorising end-of-life products. It allows, effectively, obtaining components and/or materials that can be reused or recycled with much more interesting recovery rates. To define effective disassembly and derive the economic benefits of the disassembly process, product quality uncertainty must be taken into account. In order to provide an answer to this expectation, we presented in this work a decision tool on the disassembly process planning taking into consideration the quality of the products to be disassembled. The quality 
of a product is modeled using the Remaining Usage Potential (RUP) concept. RUP models the amount of use remaining before disassembling a product (or subassembly or component). At the beginning of the operation phase of a product, RUP has a value of 1 ; a value 0 of RUP means that the product must undergo a recycling of its material. The RUP is taken as a random variable with known normal probability distribution truncated on 0 and 1.

To model this problem, a stochastic program is developed. The objective is to maximize the profit of the disassembly process. The latter is the difference between revenues of subassemblies and components and costs of the disassembly tasks. Subassemblies and components revenues are defined as functions of the RUP. All the possible disassembly alternatives of a product and the precedence relationships among tasks and subassemblies/components are modeled using an and/or graph.

The developed methodology is evaluated and applied to two product examples: a pedagogical instance (ball point pen) in order to facilitate the understanding of the modeling approach and easily assimilate the interpretation of the optimization results, an industrial instance (Knorr-Bremse EBS 1 Channel Module) which represents a real case study, in the automotive part remanufacturing sector, to show the industrial applicability of the developed optimization tool.

The optimization results have shown that the profit of the disassembly process depends on the sequence and level of disassembly of the product, and that the disassembly sequence and level are themselves dependent on the states or quality of the components and subassemblies. The results also showed that the level of disassembly for the same sequence or disassembly alternative depends on the type of variation of components and subassembly revenues according to RUP.

The obtained results are promising and have shown the applicability of the developed methodology to real industrial case. The modeling process and optimization tool presented can be easily adapted for more real life cases like End of Life Vehicles (ELV) or Waste Electrical and Electronic Equipment (WEEE). Undertaking such case studies is one of our next research objectives. Finally, as in industrial reality, the processing times of disassembly tasks are characterized by a high variability. To deal with it, we investigate in our next work the case where costs of the disassembly tasks are dependent on their processing times which will be taken as random variables. 


\section{References}

\section{References}

[1] European-Commission, Europe 2020 strategy, Tech. rep. (2010).

[2] McKinsey Center for Business and Environment, Growth within: A circular economy vision for a competitive europe., Tech. rep., Ellen Macarthur Foundation (2015).

[3] The European Factories of the Future Research Association, Factories of the future: multiannual roadmap for the contractual PPP under horizon 2020., Tech. rep. (2013).

[4] M. Colledani, O. Battaïa, A decision support system to manage the quality of end-of-life products in disassembly systems, CIRP Annals 65 (1) (2016) $41-44$.

[5] D. Parker, K. Riley, S. Robinson, H. Symington, J. Tewson, K. Jansson, S. Ramkumar, D. Peck, Remanufacturing market study., Tech. rep., ERN European Remanufacturing Network (2015).

[6] N. Nasr, M. Thurston, Remanufacturing - a key enabler to sustainable product systems, in: Industrial and consumption challenges for life cycle engineering: Proceedings of the 13th CIRP International Conference on Life Cycle Engineering, Leuven, 2006, pp. 15-18.

[7] E. Schau, M. Traverso, M. Finkbeiner, Life cycle approach to sustainability assessment:a case study of remanufactured alternators, Journal of Remanufacturing 2 (5) (2012) 1 - 14 .

[8] R. Steinhilper, F. Weiland, Exploring new horizons for remanufacturing an up-to-date overview of industries, products and technologies, Procedia CIRP 29 (2015) 769 - 773, the 22nd CIRP Conference on Life Cycle Engineering.

[9] S. Rolf, Automotive service engineering and remanufacturing: New technologies and opportunities, in: 15th CIRP International Conference on Life Cycle Engineering : Applying Life Cycle Knowledge to Engineering Solutions, 2008. 
[10] J. Duflou, G. Seliger, S. Kara, Y. Umeda, A. Ometto, B. Willems, Efficiency and feasibility of product disassembly: A case-based study, CIRP Annals - Manufacturing Technology 57 (2) (2008) 583-600.

[11] L. Diez, P. Marangé, F. Mayer, E. Levrat, Maintenance as a cornerstone for the application of regeneration paradigm in systems lifecycle, CSD\&M'15, 2015.

[12] M. R. Johnson, M. H. Wang, Planning product disassembly for material recovery opportunities, International Journal of Production Research 33 (11) (1995) 3119-3142.

[13] S. M. McGovern, S. M. Gupta, The Disassembly Line, Balancing and Modeling, 2011th Edition, McGraw-Hill Companies, New York, 2011.

[14] A. K. Subramani, P. Dewhurst, Automatic generation of product disassembly sequences, Annals of the CIRP 40 (1) (1991) 115-118.

[15] M. Santochi, G. Dini, F. Failli, Computer aided disassembly planning: State of the art and perspectives, CIRP Annals - Manufacturing Technology 51 (2) (2002) 507-529.

[16] S. Kara, P. Pornprasitpol, H. Kaebernick, Selective Disassembly Sequencing: A Methodology for the Disassembly of End-of-Life Products, CIRP Annals - Manufacturing Technology 55 (1) (2006) 37-40.

[17] T. Dong, L. Zhang, R. Tong, J. Dong, A hierarchical approach to disassembly sequence planning for mechanical product, The International Journal of Advanced Manufacturing Technology $30(5-6)(2006)$ 507-520.

[18] A. Lambert, S. Gupta, Methods for optimum and near optimum disassembly sequencing, International Journal of Production Research 46 (11) (2008) 2845-2865.

[19] J. R. Peeters, P. Vanegas, J. R. Duflou, T. Mizuno, S. Fukushige, Y. Umeda, ffects of boundary conditions on the end-of-life treatment of LCD TVs, CIRP Annals - Manufacturing Technology 62 (1) (2013) 35-38. 
[20] A. J. D. Lambert, Disassembly sequencing: a survey, International Journal of Production Research 41 (2003) 3721-3759.

[21] G. Tian, Y. Liu, Q. Tian, J. Chu, Evaluation model and algorithm of product disassembly process with stochastic feature, Clean Technologies and Environmental Policy 14 (2) (2012) $345-356$.

[22] A. J. D. Lambert, Optimizing disassembly processes subjected to sequencedependent cost, Computers \& Operations Research 34 (2007) 536-551.

[23] C. B. Kalayci, S. M. Gupta, Ant colony optimization for sequence-dependent disassembly line balancing problem, Journal of Manufacturing Technology Management 24 (3) (2013) 413-427.

[24] S. A. Reveliotis, Uncertainty management in optimal disassembly planning through learningbased strategies, IIE Transactions 39 (6) (2007) 645-658.

[25] G. Matthieu, P. F. cois, A. Tchangani, Optimising end-of-life system dismantling strategy, International Journal of Production Research 50 (14) (2012) 3738-3754.

[26] M. L. Bentaha, O. Battaïa, A. Dolgui, Disassembly line balancing and sequencing under uncertainty, Procedia CIRP 15 (2014) 239-244.

[27] M. Turowski, M. Morgan, Disassembly line design with uncertainty, 2005 IEEE International Conference on Systems, Man and Cybernetics 1 (2005) 954-959.

[28] S. Agrawal, M. K. Tiwari, A collaborative ant colony algorithm to stochastic mixed-model U-shaped disassembly line balancing and sequencing problem, International Journal of Production Research 46 (6) (2006) 1405-1429.

[29] A. Aydemir-Karadag, O. Turkbey, Multi-objective optimization of stochastic disassembly line balancing with station paralleling, Computers \& Industrial Engineering 65 (3) (2013) 413-425.

[30] M. L. Bentaha, O. Battaïa, A. Dolgui, S. J. Hu, Dealing with uncertainty in disassembly line design, CIRP Annals - Manufacturing Technology 63 (1) (2014) 21 - 24. 
[31] M. L. Bentaha, O. Battaïa, A. Dolgui, Lagrangian relaxation for stochastic disassembly line balancing problem, Procedia CIRP 201417 (2014) 56-60.

[32] M. L. Bentaha, O. Battaïa, A. Dolgui, S. J. Hu, Second order conic approximation for disassembly line design with joint probabilistic constraints, European Journal of Operational Research 247 (3) (2015) 957-967.

[33] M. L. Bentaha, O. Battaïa, A. Dolgui, An exact solution approach for disassembly line balancing problem under uncertainty of the task processing times, International Journal of Production Research 53 (6) (2015) 1807-1818.

[34] M. L. Bentaha, A. Dolgui, O. Battaïa, R. J. Riggs, J. Hu, Profit-oriented partial disassembly line design: dealing with hazardous parts and task processing times uncertainty, International Journal of Production Research (2018) 1-23.

[35] M. L. Bentaha, Combinatorial design of disassembly lines under uncertainty, 4OR (2015) 1-2.

[36] A. Güngör, S. M. Gupta, A solution approach to the disassembly line balancing problem in the presence of task failures, International Journal of Production Research 39 (7) (2001) $1427-1467$.

[37] F. T. Altekin, C. Akkan, Task-failure-driven rebalancing of disassembly lines, International Journal of Production Research 50 (18) (2012) 4955-4976.

[38] M. A. Ilgin, S. M. Gupta, Environmentally conscious manufacturing and product recovery (ECMPRO): A review of the state of the art, Journal of Environmental Management 91 (3) (2010) 563-591.

[39] M. L. Bentaha, O. Battaïa, A. Dolgui, A bibliographic review of production line design and balancing under uncertainty, IFAC-PapersOnLine 48 (3) (2015) 70-75, 15th IFAC Symposium on Information Control Problems in Manufacturing INCOM 2015.

[40] E. Özceylan, C. B. Kalayci, A. Güngör, S. M. Gupta, Disassembly line balancing problem: 
a review of the state of the art and future directions, International Journal of Production Research (2018) 1-23.

[41] R. J. Riggs, O. Battaïa, S. J. Hu, Disassembly line balancing under high variety of end of life states using a joint precedence graph approach, Journal of Manufacturing Systems 37 (2015) 638 - 648, reverse Supply Chains.

[42] A. Koc, I. Sabuncuoglu, E. Erel, Two exact formulations for disassembly line balancing problems with task precedence diagram construction using an AND/OR graph, IIE Transactions $41(10)(2009)$ 866-881.

[43] H. Rinne, The Weibull Distribution: A Handbook, London: Chapman and Hall/CRC, 2008.

[44] S. J. Almalki, S. Nadarajah, Modifications of the weibull distribution: A review, Reliability Engineering \& System Safety 124 (2014) $32-55$.

[45] C. Ly, K. Tom, C. S. Byington, R. Patrick, G. J. Vachtsevanos, Fault diagnosis and failure prognosis for engineering systems: A global perspective, in: 2009 IEEE International Conference on Automation Science and Engineering, 2009, pp. 108-115.

[46] F. Camci, G. Valentine, K. Navarra, Methodologies for integration of phm systems with maintenance data, in: IEEE Aerospace Conference Proceedings, Montana, USA, 2007.

[47] S. Kumar, E. Dolev, M. Pecht, Parameter selection for health monitoring of electronic products, Microelectronics Reliability 50 (2009) 161-168.

[48] S. M. Rezvanizaniani, Z. Liu, Y. Chen, J. Lee, Review and recent advances in battery health monitoring and prognostics technologies for electric vehicle (ev) safety and mobility, Journal of Power Sources 256 (2014) 110 - 124.

[49] L. Ungurean, G. Cârstoiu, M. V. Micea, V. Groza, Battery state of health estimation: a structured review of models, methods and commercial devices, International Journal of Energy Research 41 (2) (2017) 151-181. 